\title{
Canes Venatici I cloud of galaxies seen in the $\mathrm{H} \alpha$ line
}

\author{
S. S. Kaisin and I. D. Karachentsev
}

\author{
Special Astrophysical Observatory, Russian Academy of Sciences, N. Arkhyz, KChR, 369167, Russia \\ e-mail: ikar@luna.sao.ru
}

Received 11 September 2007 / Accepted 25 September 2007

\begin{abstract}
We present results of $\mathrm{H} \alpha$ imaging for 42 galaxies in the nearby low-density cloud Canes Venatici I, populated mainly by late-type objects. Estimates of the $\mathrm{H} \alpha$ flux and integrated star formation rate $(S F R)$ are now available for all 78 known members of this scattered system, spanning a large range in luminosity, surface brightness, HI content and SFR. Distributions of the CVnI galaxies versus their $S F R$, blue absolute magnitude and total hydrogen mass, are given in comparison with those for a population of the nearby virialized group around M 81. We found no essential correlation between star formation activity in a galaxy and its density environment. The bulk of CVnI galaxies had enough time to generate their baryon mass with the observed SFR. Most of them also possess a supply of gas that is sufficient to maintain their observed SFRs during the next Hubble time.
\end{abstract}

Key words. galaxies: evolution - galaxies: ISM - galaxies: dwarf

\section{Introduction}

The distribution over the sky of 500 galaxies of the Local Volume, with distances within $10 \mathrm{Mpc}$, shows considerable inhomogeneities due to the presence of groups and voids. Apart from several virialized groups, like the group around the galaxy M 81, an amorphous association of nearby galaxies in the Canes Venatici constellation was noted by many authors (Karachentsev 1966; de Vaucouleurs 1975; Vennik 1984; Tully 1988). The boundaries of this association are rather uncertain. Roughly, in a circle of radius $\sim 20^{\circ}$ around the galaxy NGC 4736, there are about 80 known galaxies with $D<10 \mathrm{Mpc}$, which corresponds to a density contrast on the sky $\Delta N / N \sim 4$. Inside of this complex the distribution of galaxies is also inhomogeneous, showing some clumps that differ in their location on the sky and distances in depth. About $70 \%$ of the population of the cloud is accounted for by irregular dwarf galaxies, with masses that are obviously insufficient to keep such a system in the state of virial equilibrium. As data on distances of galaxies accumulated, a possibility appeared of studying the kinematics of the cloud in details. As was shown by Karachentsev et al. (2003), the CVnI cloud is in a state close to the free Hubble expansion, having a characteristic crossing time of about 15 Gyr.

Being a scattered system with rare interactions between galaxies, the nearby $\mathrm{CVnI}$ cloud is a unique opportunity for studying star formation processes in galaxies running independently, without a noticeable external influence. Kennicutt et al. (1989), Hoopes et al. (1999), van Zee (2000), Gil de Paz et al. (2003), James et al. (2004) and Hunter \& Elmegreen (2004) conducted observations in the $\mathrm{H} \alpha$ line of three dozen galaxies of this complex, which made it possible to determine the star formation rate $(S F R)$ in them. However, more than half of the other members of the cloud proved to be out of vision of these authors. Our task consisted in the completion of the $\mathrm{H} \alpha$-survey of the population of the CVnI cloud. The results of our observations and their primary analysis are presented in this paper.

\section{Observations and data reduction}

CCD images in the $\mathrm{H} \alpha$-line and continuum were obtained for 42 galaxies of the $\mathrm{CVnI}$ cloud during observing runs from March 2001 to May 2006. An average seeing was $1.8^{\prime \prime}$. All the observations were performed in the Special Astrophysical Observatory of the Russian Academy of Sciences with the BTA 6-m telescope equipped with the SCORPIO focal reducer (Afanasiev et al. 2005). A CCD chip of $2048 \times 2048$ pixels provides a total field of view of about $6.1^{\prime}$ with a scale of $0.18^{\prime \prime} /$ pixel. The images in $\mathrm{H} \alpha+[\mathrm{NII}]$ and the continuum were obtained by observing the galaxies though a narrow-band interference filter $\mathrm{H} \alpha(\Delta \lambda=75 \AA)$ with an effective wavelength $\lambda=6555 \AA$ and two medium-band filters for the continuous spectrum, SED607 with $\Delta \lambda=167 \AA, \lambda=6063 \AA$ and SED707 with $\Delta \lambda=207 \AA$, $\lambda=7063 \AA$, respectively. Typical exposure times for the galaxies were $2 \times 300 \mathrm{~s}$ in the continuum and $2 \times 600 \mathrm{~s}$ in $\mathrm{H} \alpha$. Since the range of radial velocities in our sample is small, we used just one $\mathrm{H} \alpha$ filter for all the observed objects. Table 1 presents a brief synopsis of these runs.

Our data reduction followed standard practice and was performed within the MIDAS package. For all the data, bias was subtracted and the images were flat-fielded by twilight flats. Cosmic particles were removed and the sky background was subtracted. The next operation was to bring all the images of a given object into coincidence. The images in the continuum were then normalized to $\mathrm{H} \alpha$ images using 5-15 field stars, and subtracted. $\mathrm{H} \alpha$ fluxes were obtained for the continuumsubtracted images, using spectrophotometric standard stars from Oke (1990) observed on the same nights as the objects. The investigation of measurement errors contributed by the continuum subtraction, flat-fielding and scatter in the zeropoints, has shown that they have typical values within $10 \%$. We did not correct $\mathrm{H} \alpha$ fluxes for the contribution of the [NII] lines, because it is likely to be small for the majority of low-luminosity galaxies in our sample. 
Table 1. The observational log. (1) - the galaxy name; (2) - the date of observation; (3) - the total exposure time in seconds; a colon means that the sky was not photometric.

\begin{tabular}{lcc}
\hline \hline Galaxy & Date & $\begin{array}{c}T_{\text {exp }} \\
(1)\end{array}$ \\
\hline U5427 & $03 / 02 / 2005$ & 1200 \\
N3344 & $04 / 02 / 2005$ & 300 \\
KK 109 & $05 / 02 / 2005$ & 1200 \\
BTS 76 & $04 / 02 / 2005$ & 1200 \\
MCG 6-27-17 & $05 / 02 / 2005$ & 1200 \\
DDO 113 & $27 / 01 / 2004$ & 1200 \\
MCG 9-20-131 & $06 / 02 / 2005$ & 1200 \\
U7298 & $06 / 02 / 2005$ & 900 \\
U7356 & $05 / 02 / 2005$ & 1200 \\
I3308 & $04 / 02 / 2005$ & 600 \\
KK 144 & $04 / 02 / 2005$ & 1200 \\
N4395 & $06 / 02 / 2005$ & 300 \\
UA 281 & $24 / 05 / 2006$ & $300:$ \\
DDO 125 & $18 / 05 / 2005$ & 1200 \\
U7584 & $04 / 02 / 2005$ & 1200 \\
KKH80 & $04 / 02 / 2005$ & 1200 \\
N4449 & $24 / 05 / 2006$ & 500 \\
DDO 127 & $05 / 02 / 2005$ & 1200 \\
U7605 & $05 / 02 / 2005$ & 1200 \\
N4460 & $06 / 02 / 2005$ & 600 \\
KK 149 & $06 / 02 / 2005$ & 1200 \\
U7639 & $06 / 02 / 2005$ & 1200 \\
KK 151 & $06 / 02 / 2005$ & 1200 \\
N4627 & $06 / 02 / 2005$ & 600 \\
N4631 & $22 / 05 / 2006$ & $900:$ \\
KK 160 & $22 / 03 / 2006$ & $1200:$ \\
DDO 147 & $05 / 02 / 2005$ & 1200 \\
KK 166 & $22 / 03 / 2006$ & $1200:$ \\
U7990 & $05 / 02 / 2005$ & 1200 \\
U8215 & $16 / 05 / 2005$ & 1200 \\
N5023 & $04 / 02 / 2005$ & 600 \\
N5195 & $05 / 05 / 2005$ & 500 \\
U8508 & $17 / 03 / 2001$ & 1200 \\
N5229 & $06 / 02 / 2005$ & 1200 \\
U8638 & $06 / 02 / 2005$ & 1200 \\
DDO 181 & $24 / 05 / 2006$ & 500 \\
Holmberg IV & $29 / 01 / 2004$ & 600 \\
U8882 & $21 / 03 / 2006$ & $1200:$ \\
KK 230 & $03 / 02 / 2005$ & 1200 \\
KKH 87 & $29 / 01 / 2004$ & 1200 \\
DDO 190 & $24 / 05 / 2006$ & 400 \\
KKR 25 & $25 / 03 / 2001$ & $600:$ \\
\hline & & \\
\hline
\end{tabular}

\section{Results}

The images of 42 galaxies that we obtained in the CVnI cloud are displayed in the form of a mosaic in Fig. 1. The left and right image of every galaxy corresponds to the sum and difference of the frames exposed in $\mathrm{H} \alpha$ and the continuum. The frame size is about $4^{\prime} \times 4^{\prime}$, the North and East directions are indicated by the arrows, the scale is denoted in the lower right angle.

Some basic properties of 78 galaxies located in the $\mathrm{CVnI}$ cloud are listed in Table 2. Table 2 also includes the data on the galaxies that have been observed by other authors. The columns of Table 2 contain the following characteristics of the cloud members, taken mainly from the Catalog of Neighboring Galaxies (CNG; Karachensev et al. 2004): (1) the galaxy name; (2) and (3) the equatorial coordinates for the epoch J2000.0; (4) morphological type in numerical code according to de Vaucouleurs et al. (1991). Column (5) represents the tidal in$\operatorname{dex}(T I)$ following from the CNG; i.e. for every galaxy " $i$ " we have found its "main disturber"(=MD), producing the highest tidal action $T I_{i}=\max \left\{\log \left(M_{k} / D_{i k}^{3}\right)\right\}+C, \quad(k=1,2 \ldots N)$, where $M_{k}$ is the total mass of any neighboring potential MD galaxy (proportional to its luminosity with $M / L_{B}=10 M_{\odot} / L_{\odot}$ ) separated from the considered galaxy by a space distance $D_{i k}$; the value of the constant $C$ is chosen so that $T I=0$, when the Keplerian cyclic period of the galaxy with respect to its MD equals the cosmic Hubble time, $T_{0}$. Therefore, positive values correspond to galaxies in groups, while negative values correspond to field galaxies. Column (6) gives the galaxy radial velocity (in $\mathrm{km} \mathrm{s}^{-1}$ ) with respect to the Local Group centroid, with the apex parameters adopted in the NASA/IPAC Extragalactic Database (NED). Column (7) gives the distance to a galaxy in megaparsecs, with allowance made for new measurements (Karachentsev et al. 2006; Tully et al. 2007). Column (8) presents the blue absolute magnitude of a galaxy with the given distance, after correction for the Galactic extinction $A_{b}$ from Schlegel et al. (1998) and the internal absorption in the galaxy, determined as $A_{\text {int }}=\left[1.6+2.8\left(\log V_{m}-2.2\right)\right] \cdot \log (a / b)$, if $V_{m}>42.7 \mathrm{~km} \mathrm{~s}^{-1}$, otherwise, $A_{\text {int }}=0$. Here, $V_{m}$ is the rotation velocity of the galaxy, corrected for the inclination, and $a / b$ is the galaxy axial ratio. Therefore, we assume the internal absorption to be dependent not only on the inclination, but also on the galaxy luminosity (Verheijen 2001). Column (9) gives the logarithm of the hydrogen mass of a galaxy, $\log \left(M_{\mathrm{HI}} / M_{\odot}\right)=$ $\log F_{\mathrm{HI}}+2 \lg D_{\mathrm{Mpc}}+5.37$, defined from its flux $F_{\mathrm{HI}}$ in the $21 \mathrm{~cm}$ line; in some dwarf spheroidal galaxies the upper limit of the flux was estimated from the observations by Huchtmeier et al. (2000). Column (10) gives the logarithm of the observed integral flux of a galaxy in the $\mathrm{H} \alpha+[\mathrm{NII}]$ lines, expressed in terms of erg cm $\mathrm{cm}^{-2} \mathrm{~s}^{-1}$. Notes indicate data sources of SFRs according to other authors. Column (11) gives the star formation rate in the galaxy on a logarithmic scale, $\operatorname{SFR}\left(M_{\odot} /\right.$ year $)=1.27 \times$ $10^{9} F_{\mathrm{c}}(\mathrm{H} \alpha) \cdot D^{2}$ (Gallagher et al. 1984), where the integral flux in the $\mathrm{H} \alpha$ line is corrected for the Galactic and internal extinction as $A(\mathrm{H} \alpha)=0.538 \cdot A_{b}$, while the galaxy distance is expressed in Mpc. Columns (12) and (13) give the dimensionless parameters $p_{*}=\log \left([S F R] \cdot T_{0} / L_{B}\right)$ and $f_{*}=\log \left(M_{\mathrm{HI}} /[S F R] \cdot T_{0}\right)$, which characterize the past and the future of the process of star formation; here $L_{B}$ denotes the total blue luminosity of the galaxy in units of solar luminosity, while $T_{0}$ is the age of the universe assumed to equal to 13.7 billion years (Spergel et al. 2003).

Here we note some properties of the galaxies that we observed, in particular, their HII pattern.

UGC 5427. This dwarf galaxy with asymmetric HII filaments is situated at the fartherst western outskirts of the CVnI cloud. Its distance, 7.1 Mpc, was estimated from the luminosity of the brightest stars.

$N G C$ 3344. This is one of the rare galaxies in the cloud with a quite regular spiral pattern. Its distance of $6.9 \mathrm{Mpc}$, determined from the radial velocity at a Hubble constant $H_{0}=$ $72 \mathrm{~km} \mathrm{~s}^{-1} \mathrm{Mpc}^{-1}$, may be considerably underestimated since the galaxy is located in the Local Velocity Anomaly zone (Tully et al. 1992).

$K K$ 109. This dwarf system of low surface brightness belongs to an intermediate type between irregular and spheroidal galaxies, like the known member of the Local Group, LGS-3. Its distance, $4.51 \mathrm{Mpc}$, was determined from the tip of the red giant branch, TRGB (Karachentsev et al. 2003). There are no massive neighbors $(T I=-0.6)$ in the vicinities of KK 109, which could cause pushing of gas from this galaxy by the ram pressure. The $\mathrm{H} \alpha$-flux shown in Table 2 for the galaxy corresponds only to the upper limit. 
S. S. Kaisin and I. D. Karachentsev: Canes Venatici I cloud of galaxies seen in the H $\alpha$ line
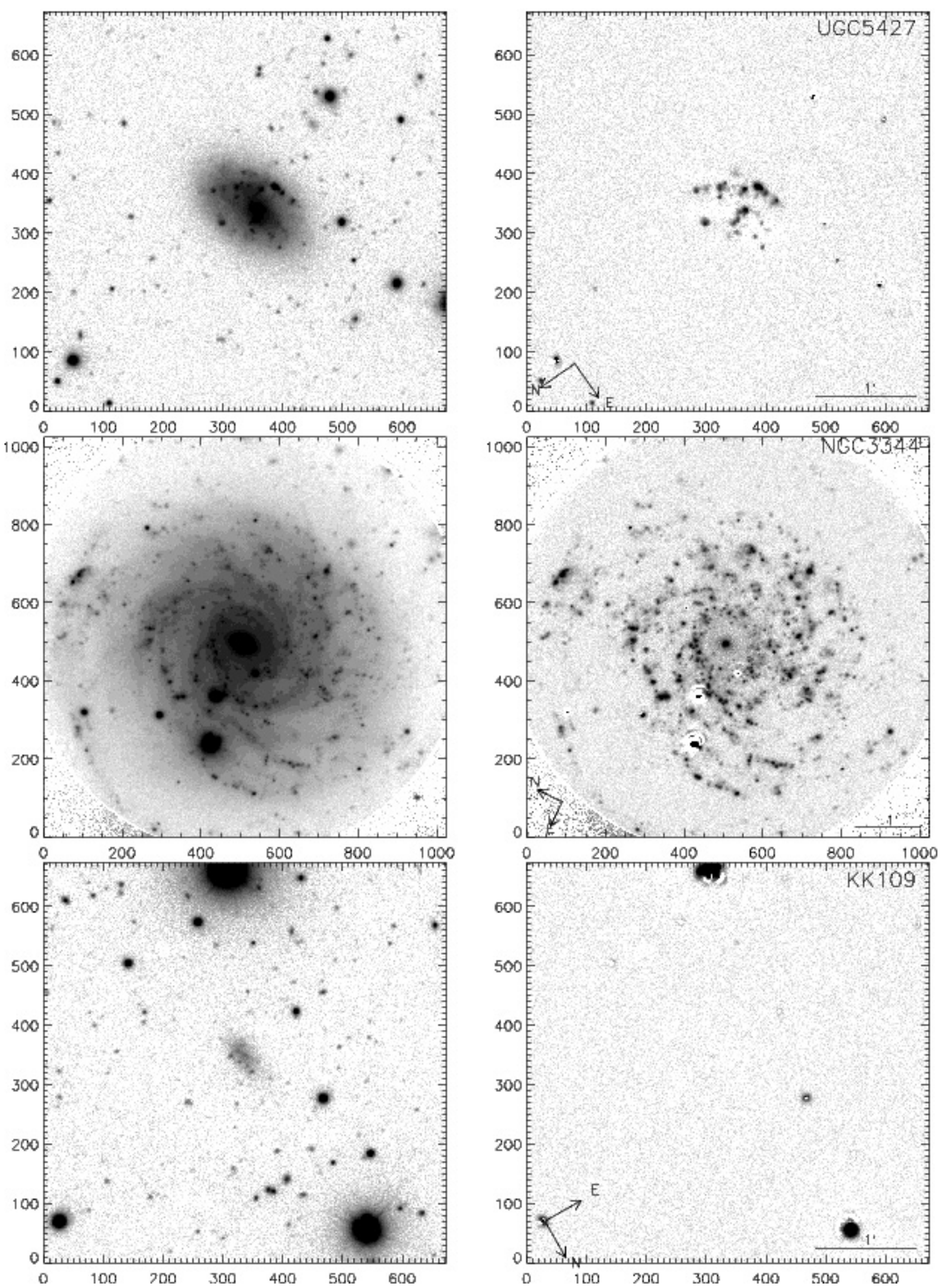

Fig. 1. The $\mathrm{H} \alpha$ (left) and the continuum-subtracted (right) images of 42 observed galaxies in the CVnI cloud. North and East are indicated by arrows. The size of the images is about $4^{\prime}$. 

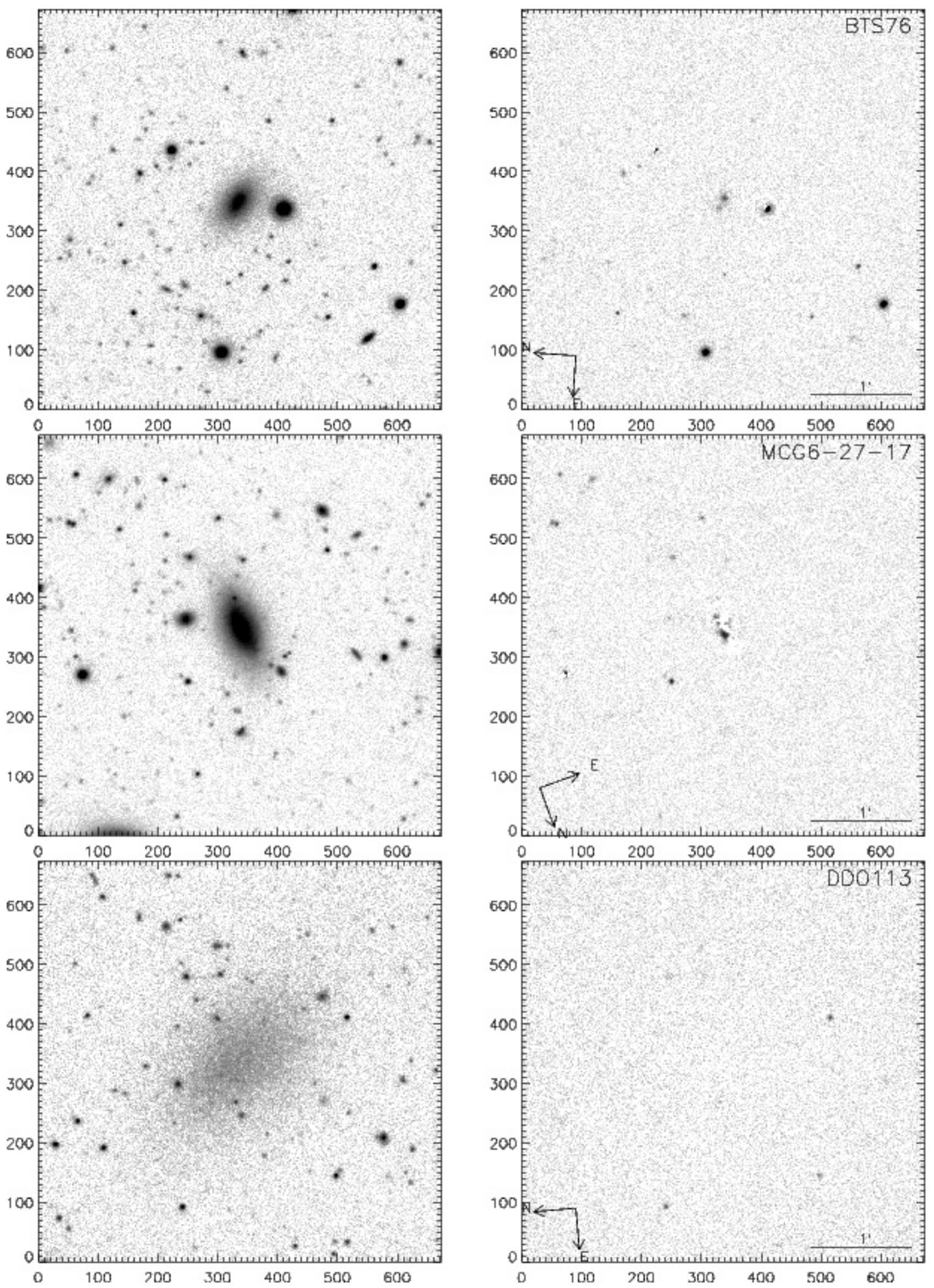

Fig. 1. continued. 
S. S. Kaisin and I. D. Karachentsev: Canes Venatici I cloud of galaxies seen in the H $\alpha$ line
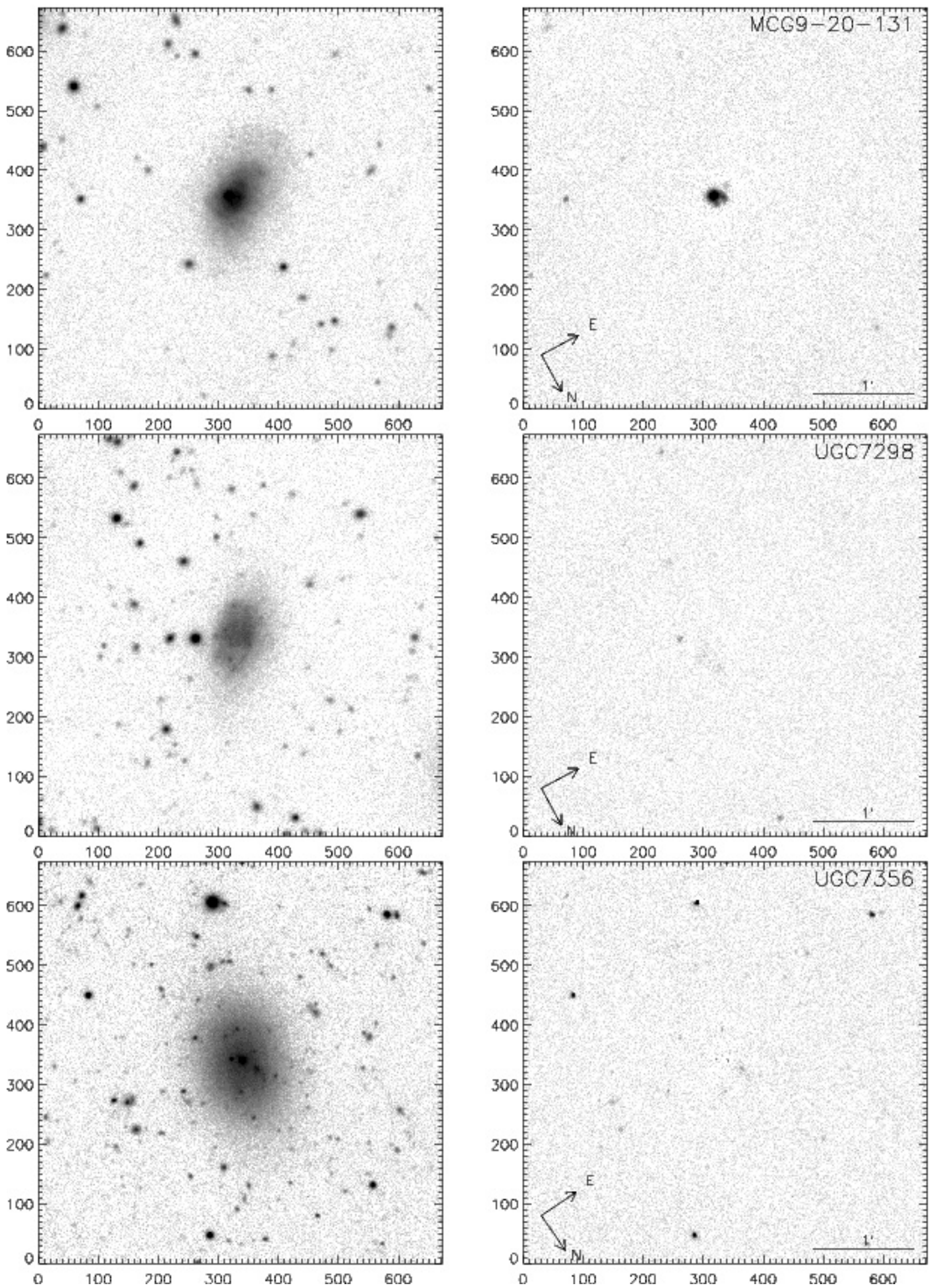

Fig. 1. continued. 

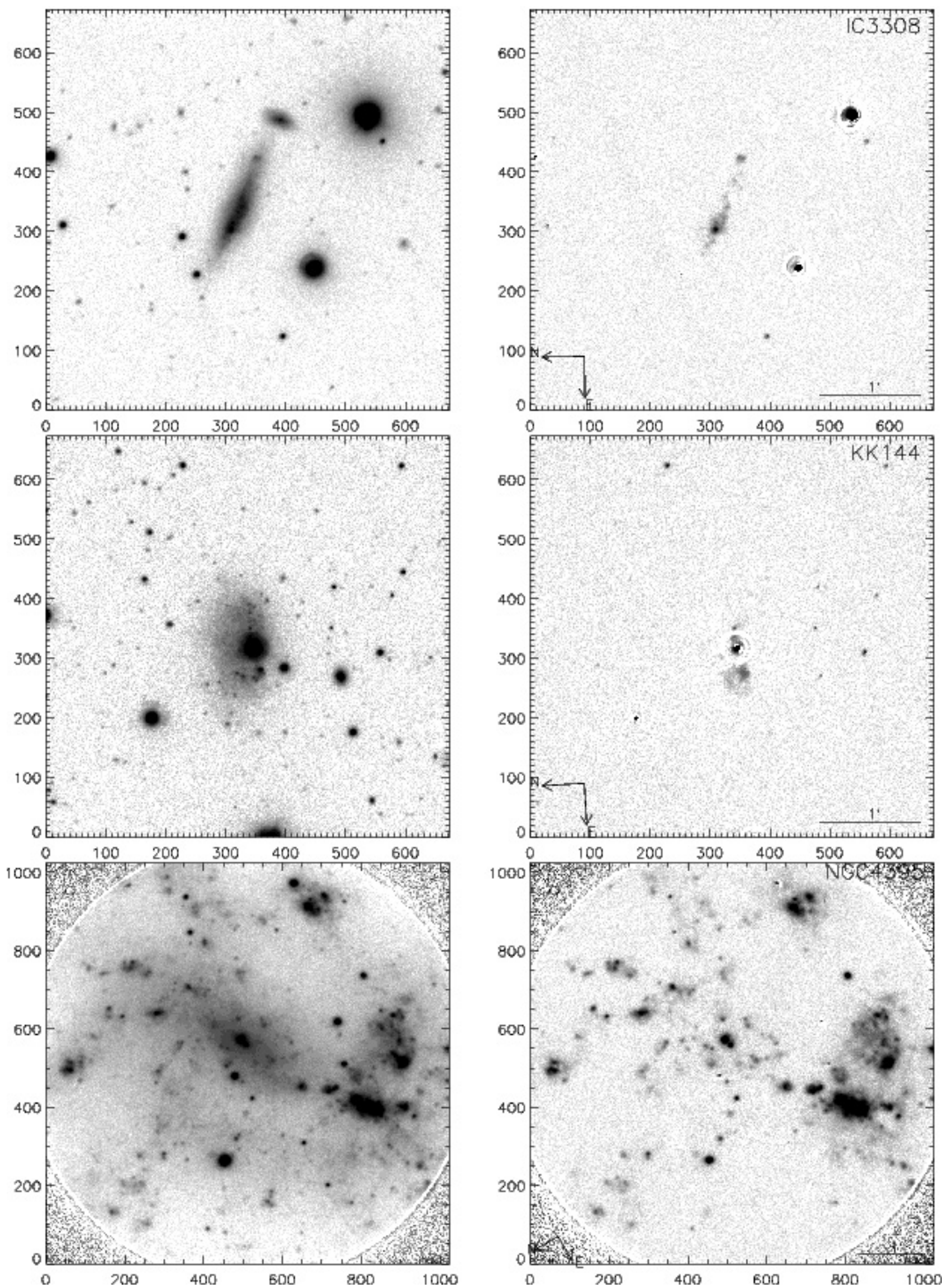

Fig. 1. continued. 
S. S. Kaisin and I. D. Karachentsev: Canes Venatici I cloud of galaxies seen in the H $\alpha$ line
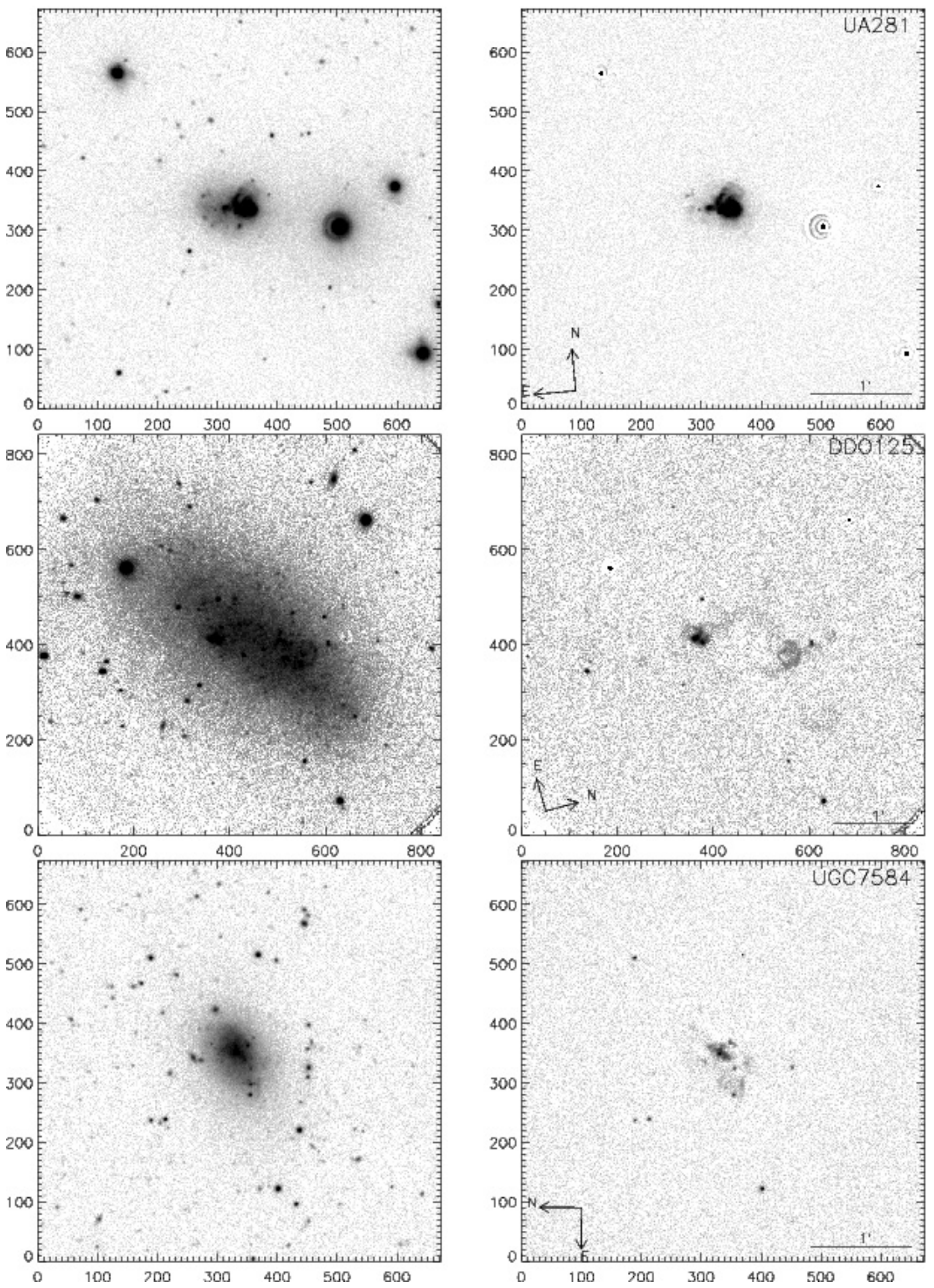

Fig. 1. continued. 

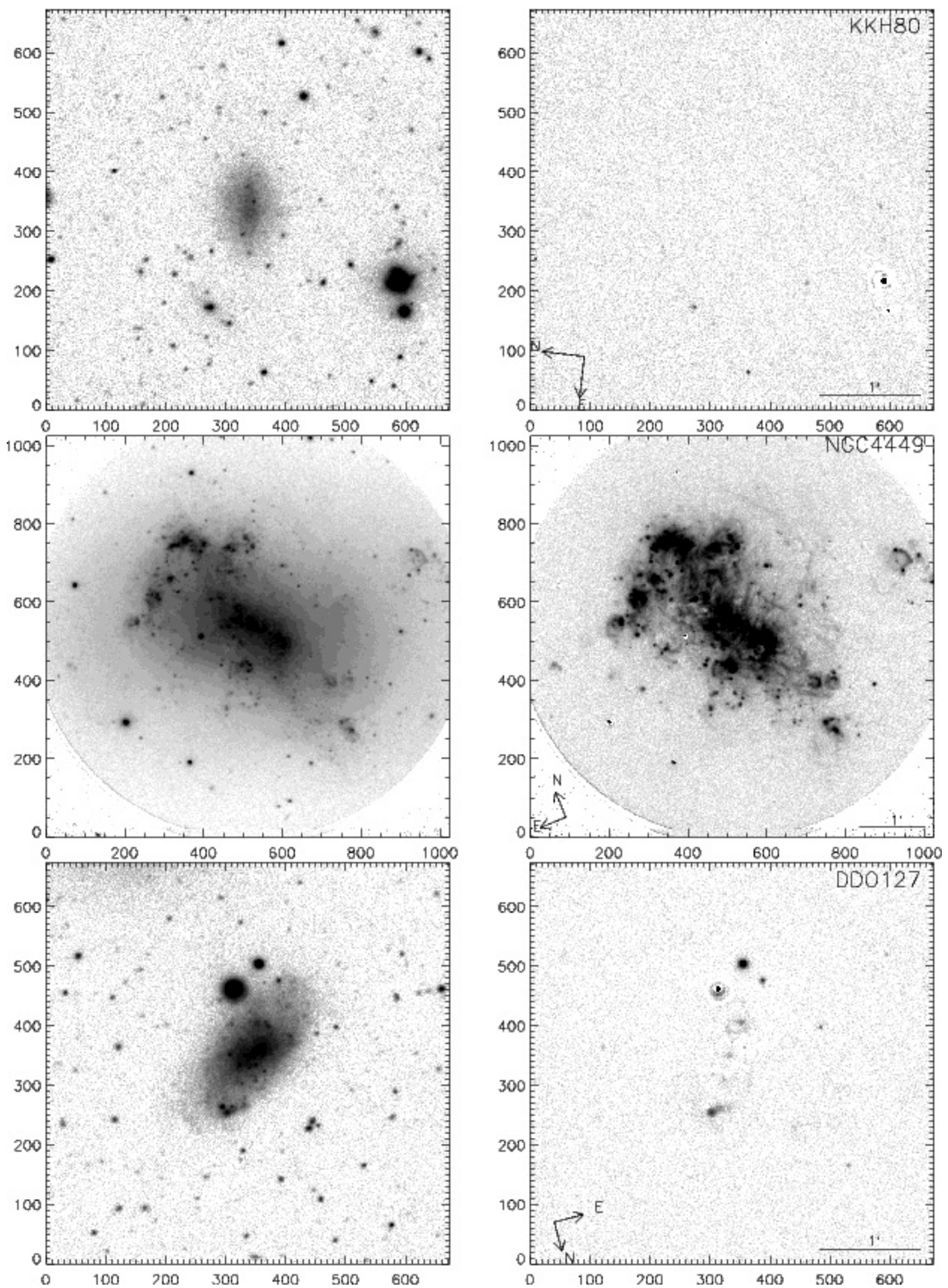

Fig. 1. continued. 
S. S. Kaisin and I. D. Karachentsev: Canes Venatici I cloud of galaxies seen in the H $\alpha$ line
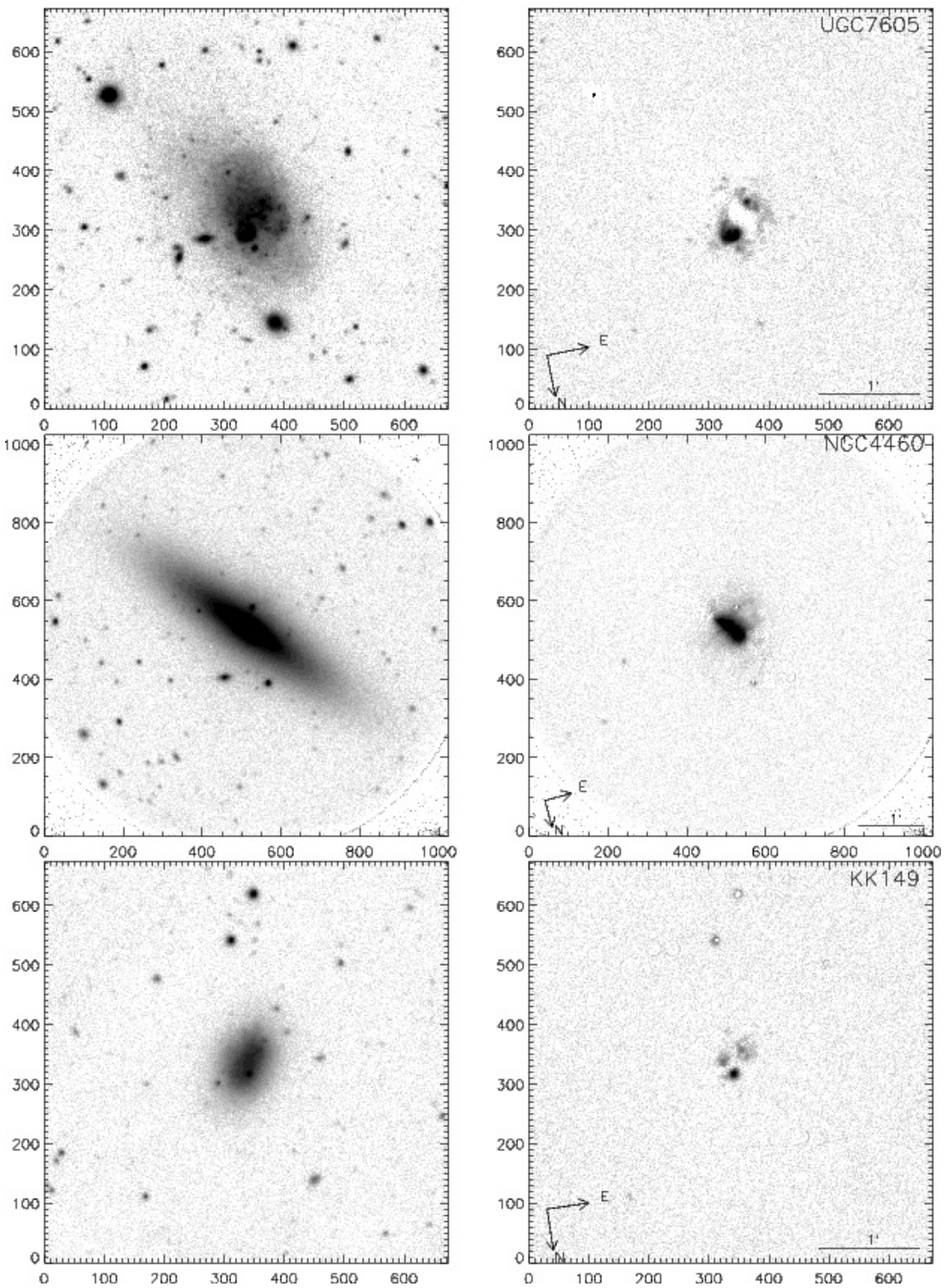

Fig. 1. continued. 

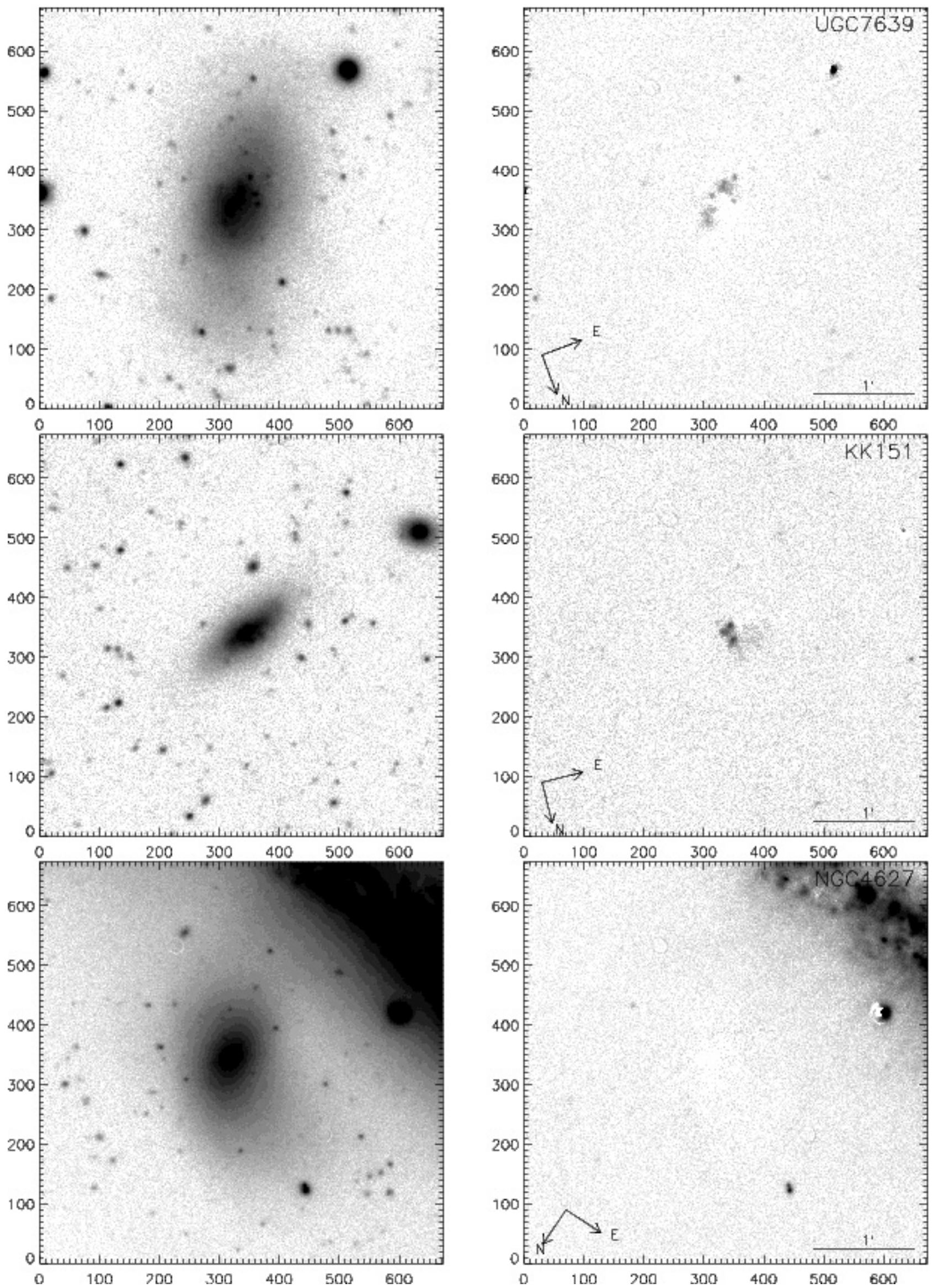

Fig. 1. continued. 

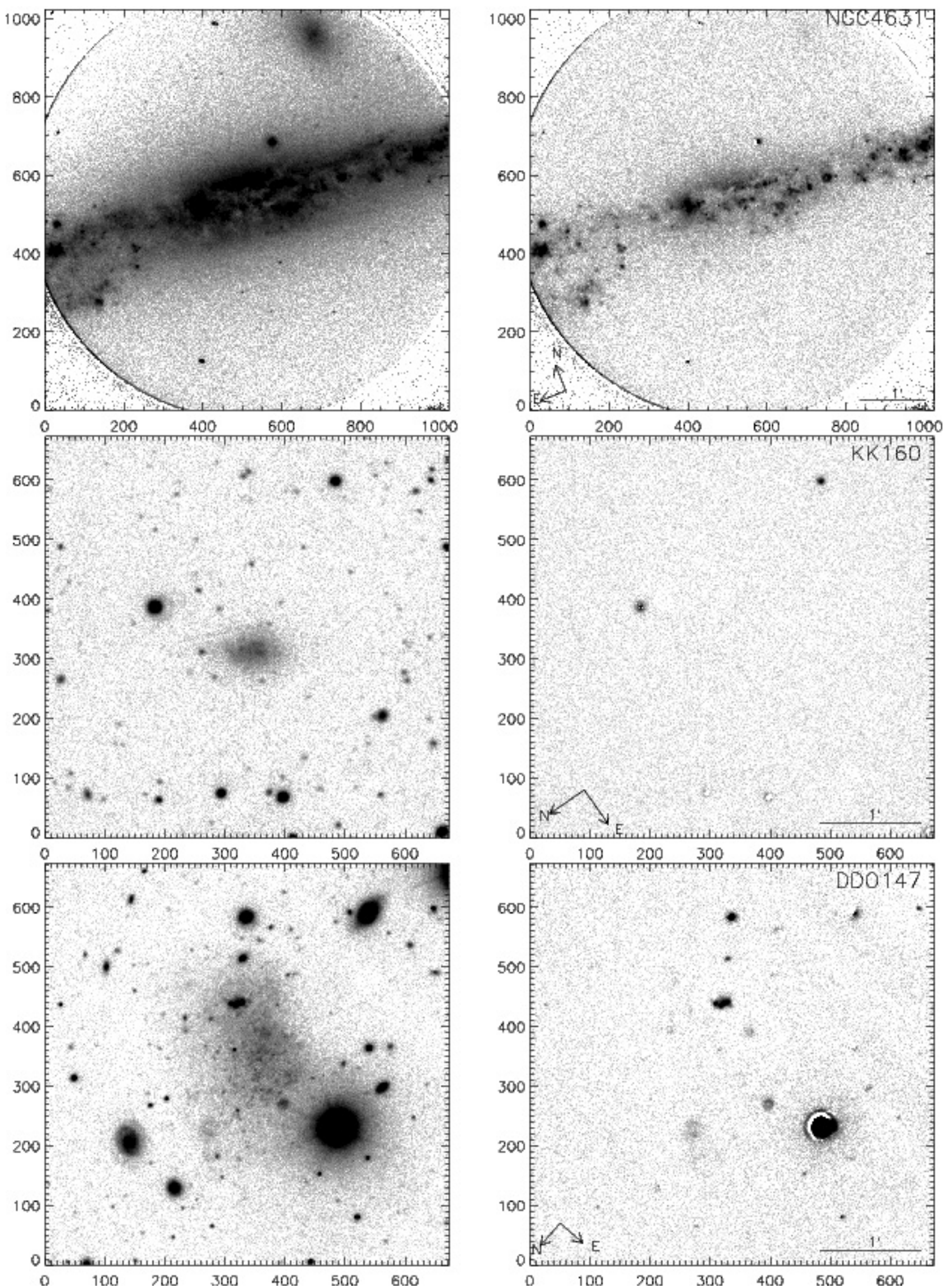

Fig. 1. continued. 

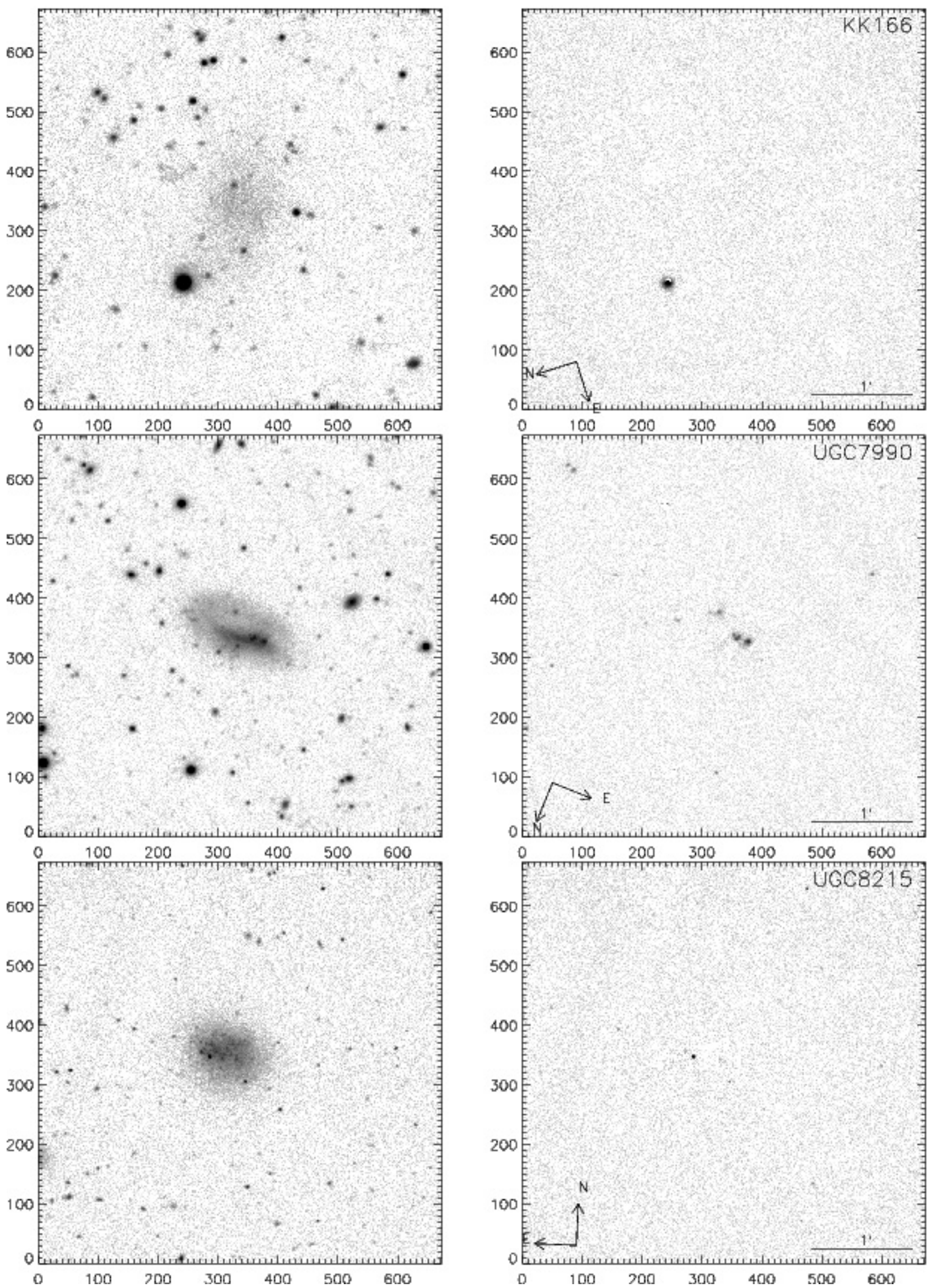

Fig. 1. continued. 

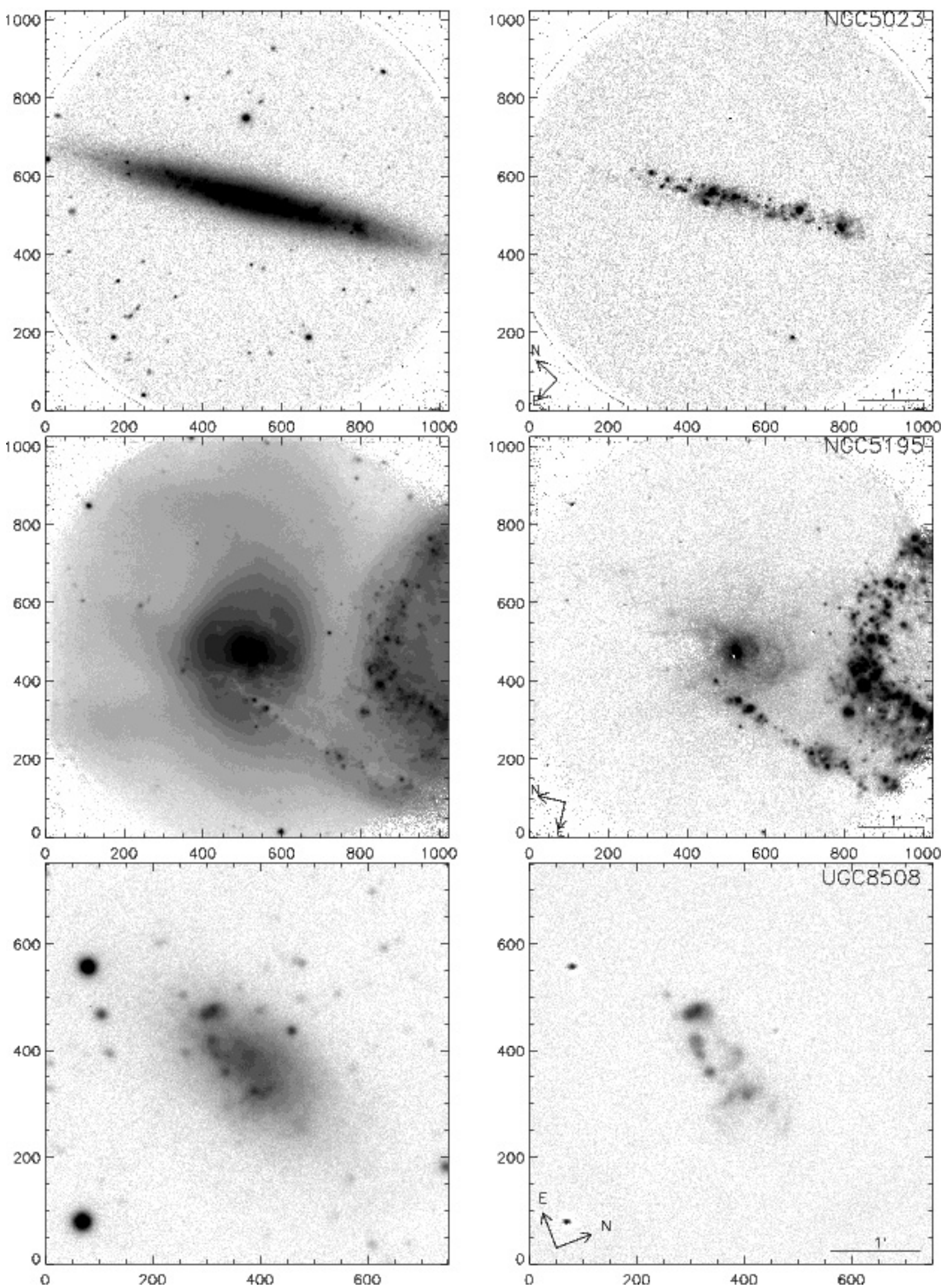

Fig. 1. continued. 

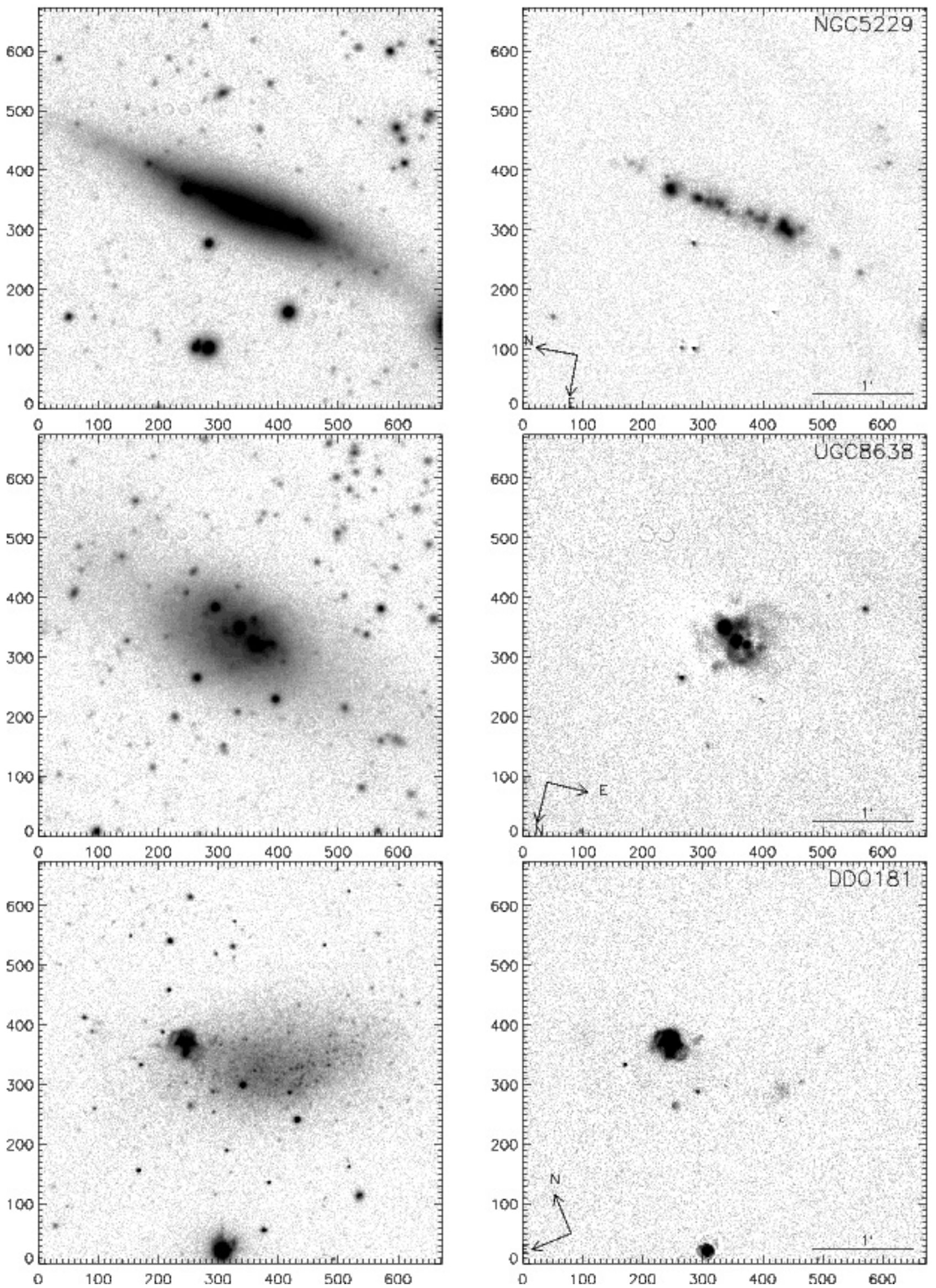

Fig. 1. continued. 
S. S. Kaisin and I. D. Karachentsev: Canes Venatici I cloud of galaxies seen in the H $\alpha$ line
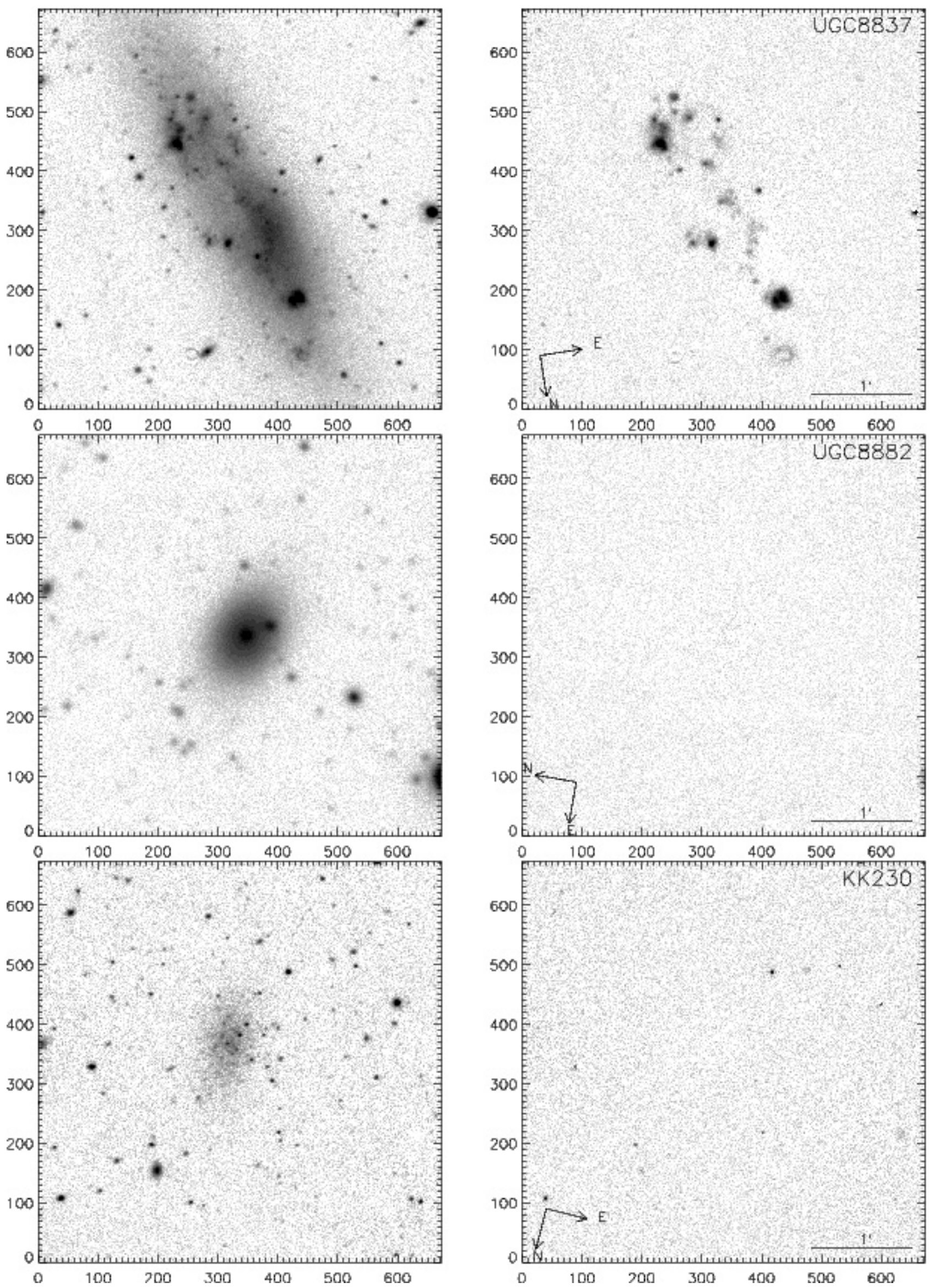

Fig. 1. continued. 

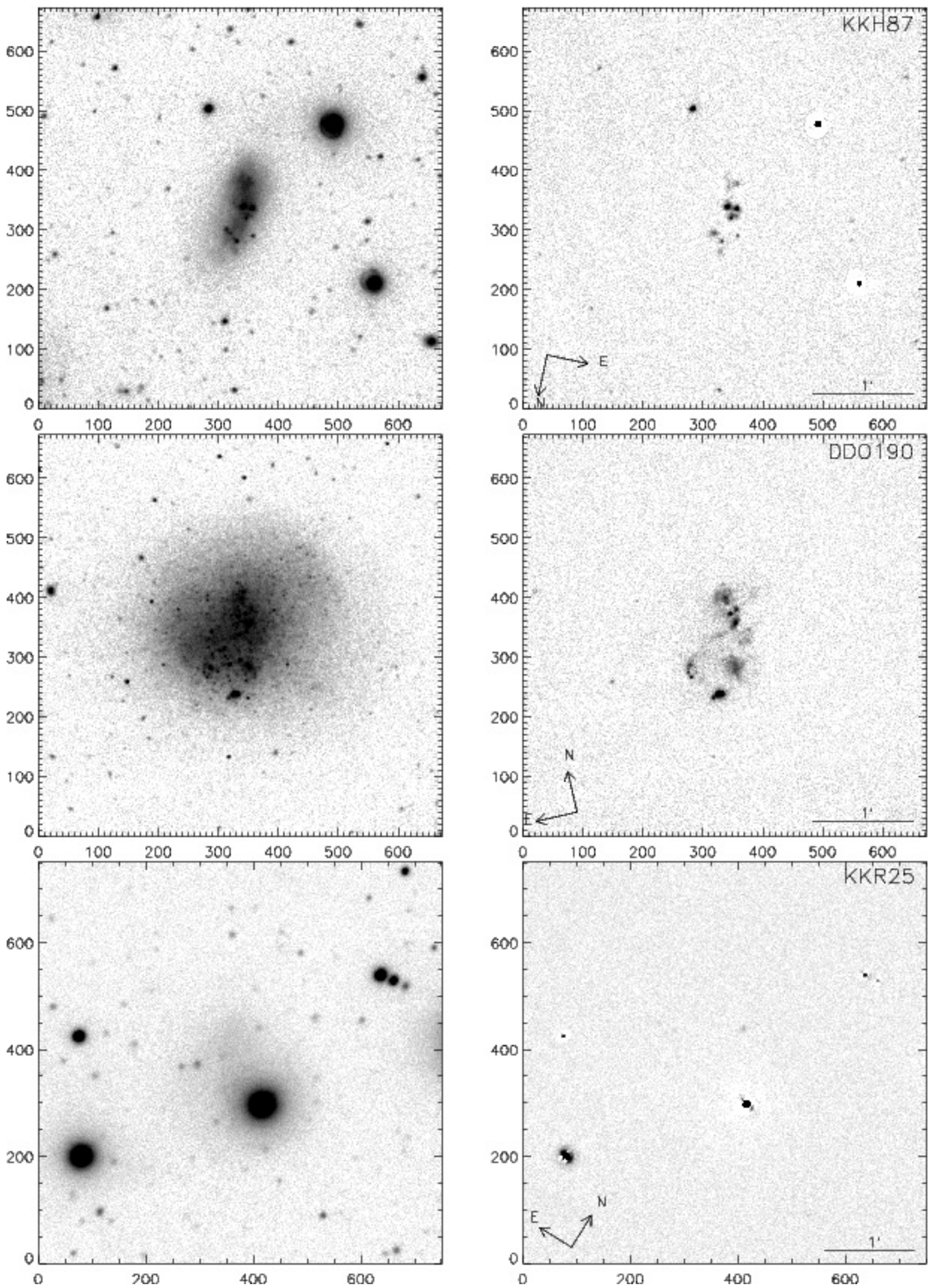

Fig. 1. continued. 
Table 2. Basic parameters of galaxies in the Canes Venatici I cloud.

\begin{tabular}{|c|c|c|c|c|c|c|c|c|c|c|c|}
\hline Name & RA (J2000) Dec & $\overline{\mathrm{T}}$ & $T I$ & $\overline{V_{\mathrm{LG}}}$ & $\begin{array}{c}D \\
\mathrm{Mpc} \\
(7)\end{array}$ & $\begin{array}{c}M_{B} \\
\text { mag } \\
(8)\end{array}$ & $\begin{array}{c}\log M_{\mathrm{HI}} \\
M_{\odot} \\
(9)\end{array}$ & $\log F$ & $\begin{array}{c}\log S F R \\
M_{\odot} \mathrm{yr}^{-1} \\
(11)\end{array}$ & (12) & (13) \\
\hline U5427 & $100441.0+292159$ & 8 & -1.2 & 424 & 7.1 & -14.48 & 7.49 & -13.03 & -2.19 & 0.00 & -0.46 \\
\hline U5672 & $102820.9+223417$ & 10 & -0.7 & 428 & 6.3 & -14.65 & 7.40 & $\mathrm{a}$ & -2.10 & 0.02 & -0.64 \\
\hline N3274 & $103217.1+274007$ & 6 & -0.3 & 461 & 6.5 & -16.16 & 8.74 & a & -1.11 & 0.41 & -0.29 \\
\hline N3344 & $104330.2+245525$ & 4 & -1.5 & 498 & 6.9 & -19.03 & 9.67 & -11.09 & -0.26 & 0.11 & -0.21 \\
\hline U6541 & $113329.1+491417$ & 10 & -0.7 & 304 & 3.89 & -13.71 & 7.03 & b & -1.68 & 0.82 & -1.43 \\
\hline N3738 & $113548.6+543122$ & 10 & -1.0 & 305 & 4.90 & -16.61 & 8.09 & a & -1.30 & 0.04 & -0.75 \\
\hline N3741 & $113606.4+451707$ & 10 & -0.8 & 264 & 3.03 & -13.13 & 8.05 & $\mathrm{a}$ & -2.28 & 0.45 & 0.19 \\
\hline KK 109 & $114711.2+434019$ & -1 & -0.6 & 241 & 4.51 & -9.73 & 6.52 & $-15.7:$ & $-5.3:$ & -1.2 : & 1.6: \\
\hline DDO 99 & $115053.0+385250$ & 10 & -0.5 & 248 & 2.64 & -13.52 & 7.71 & a & -2.48 & 0.09 & 0.05 \\
\hline BTS 76 & $115844.1+273506$ & 10 & -1.2 & 451 & 6.3 & -12.60 & 7.0: & -14.17 & -3.45 & -0.51 & 0.3: \\
\hline N4068 & $120402.4+523519$ & 10 & -1.0 & 290 & 4.31 & -15.07 & 8.13 & a & -1.65 & 0.30 & -0.36 \\
\hline MCG 627 & $120956.4+362607$ & 10 & 0.6 & 341 & 4.7 & -12.97 & 6.7: & -14.09 & -3.62 & -0.83 & 0.2 : \\
\hline N4144 & $120959.3+462726$ & 6 & 0.9 & 319 & 7.41 & -17.64 & 8.79 & $\mathrm{a}$ & -0.71 & 0.21 & -0.64 \\
\hline N4163 & $121208.9+361010$ & 9 & 1.4 & 164 & 2.96 & -13.81 & 7.29 & a & -2.83 & -0.37 & -0.02 \\
\hline N4190 & $121344.6+363760$ & 10 & 0.0 & 234 & 3.5 & -14.33 & 7.83 & $\mathrm{a}$ & -2.10 & 0.15 & -0.21 \\
\hline DDO 113 & $121457.9+361308$ & -1 & 1.6 & 283 & 2.86 & -11.67 & 6.3: & -15.0 & $-5.0:$ & -1.6: & 1.1: \\
\hline N4214 & $121538.9+361939$ & 9 & -0.7 & 295 & 2.94 & -17.19 & 8.79 & $\mathrm{c}$ & -0.64 & 0.46 & -0.71 \\
\hline MCG 920 & $121546.7+522315$ & 10 & -0.7 & 245 & 3.4 & -12.46 & 7.10 & -13.17 & -2.98 & 0.02 & -0.07 \\
\hline U7298 & $121628.6+521338$ & 10 & -0.7 & 255 & 4.21 & -12.27 & 7.31 & -14.49 & -4.12 & -1.05 & 1.29 \\
\hline N4244 & $121729.9+374827$ & 6 & -0.0 & 255 & 4.49 & -18.60 & 9.30 & d & -0.90 & -0.36 & 0.06 \\
\hline N4258 & $121857.5+471814$ & 4 & -0.7 & 507 & 7.83 & -21.25 & 9.81 & e & 0.35 & -0.17 & -0.68 \\
\hline U7356 & $121909.1+470523$ & 10 & 0.4 & 330 & 7.19 & -13.61 & 8.7: & -14.64 & -3.81 & -1.27 & 2.4: \\
\hline I3308 & $122517.9+264253$ & 7 & $-2 .:$ & 277 & 12.8 & -15.55 & 8.68 & -13.10 & -1.68 & 0.08 & 0.17 \\
\hline KK 144 & $122527.9+282857$ & 10 & -0.9 & 453 & 6.3 & -12.59 & 7.90 & -13.83 & -3.11 & -0.17 & 0.87 \\
\hline N4395 & $122549.8+333246$ & 8 & 0.1 & 315 & 4.67 & -17.88 & 9.22 & -11.04 & -0.56 & 0.27 & -0.36 \\
\hline UA 281 & $122616.0+482931$ & 9 & -0.8 & 349 & 5.43 & -13.86 & 7.84 & -11.80 & -1.21 & 1.22 & -1.09 \\
\hline DDO 126 & $122705.1+370833$ & 10 & 0.1 & 231 & 4.87 & -14.38 & 8.20 & a & -1.91 & 0.32 & -0.03 \\
\hline DDO 125 & $122741.8+432938$ & 10 & -0.9 & 240 & 2.54 & -14.16 & 7.52 & -12.67 & -2.74 & -0.42 & 0.12 \\
\hline U7584 & $122802.9+223522$ & 9 & -0.1 & 545 & 7.6 & -13.30 & 7.59 & -13.39 & -2.50 & 0.16 & -0.05 \\
\hline KKH 80 & $122805.4+221727$ & 10 & -0.1 & 542 & 7.5 & -12.47 & 7.02 & $-15.4:$ & -4.5 : & -1.5 & 1.4: \\
\hline N4449 & $122811.2+440540$ & 9 & -0.0 & 249 & 4.21 & -18.27 & 9.52 & -10.43 & -0.03 & 0.64 & -0.59 \\
\hline DDO 127 & $122828.5+371400$ & 9 & -0.7 & 291 & 6.9 & -14.28 & 8.15 & -13.64 & -2.84 & -0.57 & 0.85 \\
\hline U7605 & $122839.0+354305$ & 10 & 0.7 & 317 & 4.43 & -13.53 & 7.42 & -12.90 & -2.49 & 0.08 & -0.23 \\
\hline N4460 & $122845.8+445152$ & 1 & -0.7 & 542 & 9.59 & -17.89 & 7.65 & -11.72 & -0.59 & 0.23 & -1.90 \\
\hline KK 149 & $122852.3+421040$ & 10 & -0.8 & 446 & 6.2 & -14.06 & 7.43 & -13.39 & -2.68 & -0.32 & -0.03 \\
\hline U7639 & $122953.0+473148$ & 10 & 0.4 & 446 & 7.1 & -15.18 & 7.79 & -13.64 & -2.82 & -0.91 & 0.47 \\
\hline KK 151 & $123023.8+425405$ & 9 & -0.4 & 479 & 6.7 & -13.41 & 7.32 & -13.60 & -2.83 & -0.21 & 0.01 \\
\hline DDO 133 & $123253.0+313221$ & 10 & -1.1 & 321 & 6.1 & -15.47 & 8.58 & $\mathrm{c}$ & -1.53 & 0.26 & -0.03 \\
\hline Arp 211 & $123721.3+384443$ & 10 & -0.7 & 484 & 6.70 & -13.47 & 7.13 & b & -2.46 & 0.13 & -0.55 \\
\hline UA 292 & $123840.0+324600$ & 10 & -0.3 & 306 & 5.0 & -12.47 & 7.93 & $\mathrm{f}$ & -2.19 & 0.80 & -0.02 \\
\hline N4627 & $124159.7+323425$ & -3 & $-1.4:$ & 541 & 9.38 & -16.87 & 7.6: & -15.0 & -3.9 : & $-2.7:$ & 1.4: \\
\hline N4631 & $124208.0+323229$ & 7 & $-1.1:$ & 605 & 7.66 & -19.74 & 10.11 & -11.04 & 0.37 & 0.45 & -0.40 \\
\hline I3687 & $124215.1+383007$ & 10 & 1.1 & 385 & 4.57 & -14.64 & 8.03 & a & -2.05 & 0.07 & -0.06 \\
\hline KK 160 & $124357.4+433941$ & 10 & 1.0 & 346 & 4.8 & -11.52 & 6.58 & $-15.1:$ & -4.6 & $-1.3:$ & 1.1: \\
\hline DDO 147 & $124659.8+362835$ & 10 & -1.4 & 351 & 9.9 & -14.94 & 8.62 & -13.26 & -2.15 & -0.15 & 0.63 \\
\hline KK 166 & $124913.3+353645$ & -3 & 0.3 & & 4.74 & -10.82 & 6.4: & -15.15 : & $-4.7:$ & -1.0 & 0.9: \\
\hline U7990 & $125027.0+282107$ & 10 & $-2 .:$ & 495 & 20.9 & -15.46 & 8.38 & -14.01 & -2.25 & -0.45 & 0.49 \\
\hline N4736 & $125053.5+410710$ & 2 & -0.5 & 353 & 4.66 & -19.83 & 8.68 & $\mathrm{e}$ & -0.19 & -0.14 & -1.27 \\
\hline DDO 154 & $125405.2+270855$ & 10 & -1.1 & 355 & 3.91 & -13.87 & 8.72 & a & -2.08 & 0.35 & 0.66 \\
\hline N4826 & $125644.2+214105$ & 2 & -0.6 & 364 & 4.44 & -19.77 & 8.42 & $\mathrm{~g}$ & -0.54 & -0.47 & -1.18 \\
\hline I4182 & $130549.3+373621$ & 9 & 0.6 & 356 & 4.70 & -16.40 & 8.53 & a & -1.61 & -0.19 & 0.00 \\
\hline U8215 & $130803.6+464941$ & 10 & -0.3 & 297 & 4.55 & -12.30 & 7.32 & -13.77 & -3.34 & -0.28 & 0.52 \\
\hline N5023 & $131211.9+440219$ & 6 & -1.6 & 476 & 6.61 & -17.12 & 8.70 & -12.03 & -1.11 & 0.02 & -0.33 \\
\hline DDO 167 & $131322.8+461911$ & 10 & 0.0 & 243 & 4.19 & -12.70 & 7.27 & $\mathrm{c}$ & -2.64 & 0.26 & -0.23 \\
\hline DDO 168 & $131428.6+455510$ & 10 & -0.0 & 273 & 4.33 & -15.28 & 8.42 & $\mathrm{a}$ & -1.83 & 0.04 & 0.11 \\
\hline
\end{tabular}

BTS 76 and MGC 6-27-17. These are two relatively compact bluish galaxies, the shape of which is not notable for great irregularity. They have not been resolved into stars yet, and the distances to them are estimated from their radial velocities. Compact emission knots are seen in the central parts of both galaxies.
DDO $113=K D G 90$. This is a dwarf system of regular shape and low surface brightness. It has been resolved into stars with the Hubble Space Telescope (HST). Judging by its CM-diagram, DDO 113 contains an old stellar population and can be classified as dSph. We have not found in it either HII regions or diffuse $\mathrm{H} \alpha$ emission. The measurement of its HI-flux is hampered by 
Table 2. continued.

\begin{tabular}{|c|c|c|c|c|c|c|c|c|c|c|c|}
\hline Name & $\overline{\mathrm{RA}(\mathrm{J} 2000) \mathrm{Dec}}$ & (4) & $\overline{T I}$ & $\overline{\overline{V_{\mathrm{LG}}}}$ & $\begin{array}{c}D \\
\mathrm{Mpc} \\
(7)\end{array}$ & $\begin{array}{c}M_{B} \\
\text { mag } \\
(8)\end{array}$ & $\begin{array}{c}\log M_{\mathrm{HI}} \\
M_{\odot} \\
(9)\end{array}$ & $\overline{\log F}$ & $\begin{array}{c}\log S F R \\
M_{\odot} \mathrm{yr}^{-1} \\
(11)\end{array}$ & (12) & (13) \\
\hline DDO 169 & $131530.7+472947$ & 10 & -0.2 & 345 & 4.23 & -13.71 & 7.81 & $a$ & -2.40 & 0.10 & 0.07 \\
\hline N5204 & $36.4+582504$ & 9 & -1.1 & 341 & 4.65 & -16.75 & 8.76 & c & -1 & 4 & -0.34 \\
\hline N5194 & $956.0+4$ & 5 & 4.1 & 5 & 8.0 & -21.34 & 9.52 & g & 56 & 0 & -1.18 \\
\hline $\mathrm{N}$ & $58.7+4$ & -1 & 5.0 & 558 & 8.02 & -19.22 & 8.18 & -11.33 & -0.38 & -0.09 & -1.58 \\
\hline U8 & $44.4+$ & 10 & -1.0 & 186 & 2.56 & -12.98 & 7.34 & -12.51 & -2.57 & 0.22 & -0.23 \\
\hline N5229 & $402.9+475455$ & 7 & -0.6 & 460 & 5.1 & -14.60 & 8.18 & -12.46 & -1.86 & 0.28 & -0.10 \\
\hline N5238 & $442.7+513650$ & 9 & -0.9 & 345 & 4.24 & -14.64 & 7.27 & $\mathrm{a}$ & -2.04 & 0.08 & -0.83 \\
\hline U8638 & $919.4+244633$ & 9 & -1.3 & 273 & 4.27 & -13.77 & 7.22 & -12.68 & -2.30 & 0.17 & -0.62 \\
\hline DDO 181 & $953.8+404421$ & 10 & -1.3 & 272 & 3.02 & -12.97 & 7.39 & -12.58 & -2.51 & 0.28 & -0.24 \\
\hline DD & $051.1+3$ & 10 & -1.2 & 257 & 3.18 & -13.13 & 7.36 & 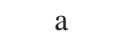 & -3.04 & -0.31 & 0.26 \\
\hline HolmIV & $5.1+5$ & 10 & 0.6 & 276 & 6.83 & -15.48 & 8.34 & -12.40 & -1.61 & 0.18 & -0.19 \\
\hline & $3.7+3$ & 10 & -1.4 & 285 & 3.12 & -12.37 & 7.14 & $e^{-1}$ & -3.06 & -0.03 & 0.06 \\
\hline U8882 & 603 & -1 & 0.0 & & 8.3 & -13.64 & 7.2: & -15.0 & $-4.1:$ & -1.6 & 1.2: \\
\hline M101 & $.8+542102$ & 6 & 0.6 & 379 & 7.38 & -21.23 & 10.36 & e & 0.74 & 0.23 & -0.52 \\
\hline N5474 & & 8 & 2.0 & 413 & 7.2 & -17.74 & 12 & $\mathrm{a}$ & -0.82 & 6 & -0.20 \\
\hline N5477 & & 9 & 1.4 & 443 & 7.7 & -15.29 & 26 & $\mathrm{a}$ & -1.27 & 59 & -0.61 \\
\hline $\mathrm{Kl}$ & 7 & 10 & -1.0 & 126 & 1.92 & -9.57 & 35 & $-15.32:$ & $-5.6:$ & -1.5 : & 1.85: \\
\hline & & 10 & 1 & 473 & 7. & -13.30 & 7.64 & -13.42 & -2.57 & 9 & 0.07 \\
\hline DD & & 10 & -1.2 & 172 & 2.28 & -12.51 & 7.16 & a & -3.57 & -0.59 & 0.59 \\
\hline & & 4 & -0.8 & 459 & 5. & -17.82 & 8.98 & $\mathrm{a}$ & -0.85 & 0.00 & -0.31 \\
\hline & $5+1$ & 10 & -1.3 & 263 & 2.79 & -14.18 & 7.63 & -12.53 & -2.52 & -0.21 & 0.01 \\
\hline DDO 19 & 524 & 10 & -0.0 & 385 & 8.0 & -15.05 & 8.00 & $\mathrm{a}$ & -2.18 & -0.22 & 0.04 \\
\hline KKR 25 & $161347.6+542216$ & -1 & -0.7 & 68: & 1.86 & -9.94 & 4.9: & $-14.64:$ & -5.0 & -1.0 & -0.2 : \\
\hline
\end{tabular}

Notes.

a - James et al. (2004); b - Gil de Paz et al. (2003); c - Hunter \& Elmegreen (2004); d - Strickland et al. (2004); e - Kennicutt et al. (1989); $\mathrm{f}$ - van Zee (2000); g - Young et al. (1996).

close proximity $\left(\sim 10^{\prime}\right)$ with the bright Im-galaxy NGC 4214, of which DDO 113 is evidently a close companion.

$M G C$ 9-20-131 = CGCG 269-049. In the central part of this dIrr galaxy, a bright HII region is seen. Together with UGC 7298, it is likely to constitute a physical pair of dwarf galaxies investigated in the HI line with the Giant Metrewave Radio Telescope (GMRT) (Begum et al. 2006).

$U G C 7298$ and $U G C 7356$. These are irregular galaxies with a low content of HI. Their distances, 4.21 Mpc (UGC 7298) and 7.19 Mpc (UGC 7356), have recently been measured by Karachentsev et al. (2003) and Tully et al. (2007). Some faint emission regions are seen in both galaxies. It is possible that, the high HI-flux from UGC 7356 is due to contamination from the bright neighboring spiral NGC 4258, since the anomalously high ratio $\left(M_{\mathrm{HI}} / L_{B}=13.5 M_{\odot} / L_{\odot}\right)$ looks inconsistent with the morphology of this galaxy.

IC $3308=U G C$ 7505. From the Tully-Fisher relation with the HI line width $W_{50}=128 \mathrm{~km} \mathrm{~s}^{-1}$, we derived the galaxy distance to be $12.8 \mathrm{Mpc}$.

$K K 144$. The measurement of $\mathrm{H} \alpha$-flux is impeded because of a bright star projected near the galaxy center.

$N G C$ 4395. This is a Seyfert 1 type galaxy having a starlike nucleus, which is lost among bright emission regions scattered over the disk. The integrated $\mathrm{H} \alpha$-flux in NGC 4395, as well as in the galaxies NGC 4449 and NGC 4631, was corrected for the incomplete field of view under the assumption that the $\mathrm{H} \alpha$-emission in these galaxies is distributed in the same way as the blue luminosity.

UGCA 281=Mkn 209. On the western side of this blue compact dwarf galaxy (BCD), there is a very bright emission knot with a short arc. Judging by the distance, $5.43 \mathrm{Mpc}$, measured by Tully et al. (2007) with HST, this compact starburst galaxy has no close neighbors and is quite appropriate to be called "intergalactic HII region", a name given by Sargent \& Searle (1970). We note some inconsistency in the integrated blue magnitudes for this galaxy, i.e. Papaderos et al. (1996) obtained $B_{T}=14.84$, while Makarova et al. (1997) and Gil de Paz et al. (2003) obtained 15.14 and $14.15 \mathrm{mag}$, respectively. We observed UGCA 281 with the 6-m telescope in the $B, V, R$ bands and obtained $B_{T}=14.87 \pm 0.05$. This magnitude measured by Sharina is presented in Table 2.

DDO $125=U G C 7577$. The diffuse emission regions in this dIrr galaxy form a knotty filament extending as far as the northern side of the galaxy.

$U G C$ 7584. Together with diffuse emission regions, zones of noticeable internal absorption are seen in the galaxy, which is not typical of dwarf galaxies.

$K K H$ 80. This is a galaxy of low surface brightness and of quite regular shape. In Table 2, only the upper limit of its $\mathrm{H} \alpha-$ flux is presented. Most likely, KKH 80 belongs to the transition dIrr/dSph type.

$N G C 4449$. The bright galaxy of Magellanic type reveals numerous powerful starburst sites scattered over the whole disk. Its emission filament structure has been described in more detail by Hunter \& Gallagher (1992).

$N G C 4460$. This is a lenticular galaxy without signs of spiral structure. Surprisingly, we find a compact emission disk in its core, from which diffuse emission protuberances originated, along the minor axis. Using the surface brighness fluctuations, Tonry et al. (2001) derived its distance to be $9.59 \mathrm{Mpc}$.

$N G C 4627$ and NGC 4631. This is a close-in-projection pair of $\mathrm{dE}$ and $\mathrm{Sd}$ galaxies with distances of $9.38 \mathrm{Mpc}$ and $7.66 \mathrm{Mpc}$, respectively. The elliptical component shows weak peripheral distortion, which is likely to give grounds to include this pair into the catalog of peculiar systems (Arp 1966). However, with errors in the estimation of the distances to the galaxies of $\sim 15 \%$, 
Table 3. Comparison of SFR estimates for the CVnI galaxies. The index " $6 \mathrm{~m}$ " at $\log [S F R]$ corresponds to our measurements, while the index "oth" corresponds to other sources indicated in the last column.

\begin{tabular}{llcrl}
\hline \hline Galaxy & $\log (\text { SFR })_{6 m}$ & $\log (\text { SFR })_{\text {oth }}$ & \multicolumn{1}{c}{$\Delta$} & Source \\
\hline NGC 4395 & -0.56 & -0.59 & 0.03 & Kennicutt et al. (1989) \\
UGCA 281 & -1.21 & -1.19 & -0.02 & Gil de Paz et al. (2003) \\
DDO 125 & -2.74 & -2.72 & -0.02 & Hunter \& Elmegreen (2004) \\
NGC 4449 & -0.03 & 0.01 & -0.04 & Kennicutt et al. (1989) \\
NGC 4631 & $0.37:$ & 0.42 & -0.05 & Hoopes et al. (1999) \\
NGC 4631 & $0.37:$ & 0.41 & -0.04 & Kennicutt et al. (1989) \\
UGC 8508 & -2.57 & -2.51 & -0.06 & James et al. (2004) \\
DDO 181 & -2.51 & -2.47 & -0.04 & van Zee (2000) \\
HolmbergIV & -1.61 & -1.57 & -0.04 & James et al. (2004) \\
DDO 190 & -2.52 & -2.43 & -0.09 & James et al. (2004) \\
DDO 190 & -2.52 & -2.56 & 0.04 & van Zee (2000) \\
\hline
\end{tabular}

they may constitute a physical pair of the M32+M31 type, in which the $\mathrm{dE}$ component lost its gas because of the influence of a close massive neighbor.

$K K 160$. This is a galaxy of the transition dIrr/dSph type with weak emission in $\mathrm{HI}$, but without visible emission in the $\mathrm{H} \alpha$ line.

DDO $147=U G C$ 7946. This dIrr galaxy has several emission regions of different degrees of compactness. Its distance, 9.9 Mpc, is estimated from the brightest stars and needs refinement.

$K K$ 166. This is a dSph galaxy of very low surface brightness, for which only upper limits of the fluxes in $\mathrm{HI}$ and $\mathrm{H} \alpha$ are available. This is one of the faintest $\left(M_{B}=-10.82\right)$ known members of the CVnI cloud.

UGC 7990. The distance to this galaxy (20.9 Mpc), derived from the Tully-Fisher relation, is greatly different from the estimate made from its radial velocity $(6.9 \mathrm{Mpc})$. The galaxy has not been resolved into stars yet.

$U G C 8215$. To the east of this dIrr galaxy there is a nearly star-like emission knot.

$N G C 5023$. This isolated late-type spiral galaxy, seen almost edge-on, was observed with ACS at HST by Seth et al. (2005), who estimated its distance to be $6.61 \mathrm{Mpc}$, based on the TRGB luminosity. All visible $\mathrm{H} \alpha$ emission from NGC 5023 is concentrated in its disk, without signs of extra-planar gas motions.

NGC 5195. This is an elliptical component of the famous interacting pair M51. Its diffuse $\mathrm{H} \alpha$ emission is distributed over the galaxy body non-uniformly. A considerable part of the $\mathrm{H} \alpha$-flux is concentrated in the circumnuclear region. Some uncertainty in the evaluation of the integrated flux from NGC 5195 is introduced by the HII regions of the spiral arm of NGC 5194 crossing the elliptical component of the pair.

NGC 5229. This is a late-type spiral galaxy seen almost edge-on. The edges of its disk are slightly curved to the opposite sides, giving the galaxy an "integral-like" shape.

UGC 8638. This is a compact galaxy whose main $\mathrm{H} \alpha$ emission comes from several compact HII regions near its center.

DDO $181=U G C$ 8651. The galaxy has a curved bow-like shape similar to DDO 165. The basic H $\alpha$-flux in DDO 181 is emitted from a very bright HII region at the eastern edge.

Holmberg $I V=U G C$ 8837. This is an irregular galaxy whose $\mathrm{H} \alpha$-emission is concentrated in compact HII regions without signs of a diffuse component. Judging by the distance of $6.83 \mathrm{Mpc}$ measured by Tully et al. (2007), it is a satellite of the bright spiral galaxy M101.

$U G C$ 8882. According to Rekola et al. (2005), this is a $\mathrm{dE}$ galaxy at a distance of $8.3 \mathrm{Mpc}$, with a compact nucleus and without visible emission in the lines $\mathrm{HI}$ and $\mathrm{H} \alpha$.
$K K 230$. This is an isolated dIrr galaxy of low surface brightness situated between the CVnI cloud and the Local Group at a distance of $1.92 \mathrm{Mpc}$. No signs of $\mathrm{H} \alpha$-emission are seen. The distribution of neutral hydrogen in it, with high angular resolution, has been investigated by Begum et al. (2006).

$K K H$ 87. This dIrr galaxy is a likely companion to M101. Almost all $\mathrm{H} \alpha$-flux of KKH 87 comes from compact HII regions without signs of a diffuse component.

DDO $190=U G C$ 9240. The periphery of the galaxy has quite a regular shape. Compact and diffuse emission knots occupy the central and southern areas of the galaxy. Being an isolated dwarf system, DDO 190 is an expressive example of a star formation burst not triggered by external tides.

$K K R 25$. This is an isolated dSph galaxy of very low surface brightness, near which a bright star is projected. KKR 25 is located between the CVnI cloud and the Local Group at a distance of $1.86 \mathrm{Mpc}$. Its radial velocity of $+68 \mathrm{~km} \mathrm{c}^{-1}$, measured by Huchtmeier et al. (2003), has not been corroborated by deeper observations with GMRT (Begum \& Chengalur 2005), who estimated only the upper limit of the HI flux. On the northern side of KKR 25 one can see a faint knot in $\mathrm{H} \alpha$, the nature of which can be established by spectral observations.

\section{External SFR comparison}

In order to test our photometry, we compared the data on SFR from Table 2 against previous flux measurements. We have found eleven cases in the literature where $\mathrm{H} \alpha$-fluxes in the galaxies of the $\mathrm{CVnI}$ cloud were measured by other authors. A summary of these data is presented in Table 3. The literature $\mathrm{H} \alpha$-fluxes were correcte for the external (Galactic) and internal absorption in the manner described, above and reduced to the galaxy distances given in Table 2 .

The mean offset is $\Delta \log [S F R]=-0.03 \pm 0.01$, in the sense that our study finds about $7 \%$ lower fluxes than literature studies, possibly due to the lower effective apertures used. The eleven points have an RMS scatter of 0.04 dex about the regression line. Given the above mentioned uncertainties and differences in reduction procedures, the agreement found in Table 3 seems to be generally good.

\section{Discussion}

As it has been noted above, the population of the CVnI cloud is distinguished from other nearby, but virialized, groups by two basic features: a) most galaxies in the cloud $(\sim 70 \%)$ do not actually interact with their closest neighbors, having negative tidal indices; b) the majority of the cloud objects $(\sim 70 \%)$ are galaxies 


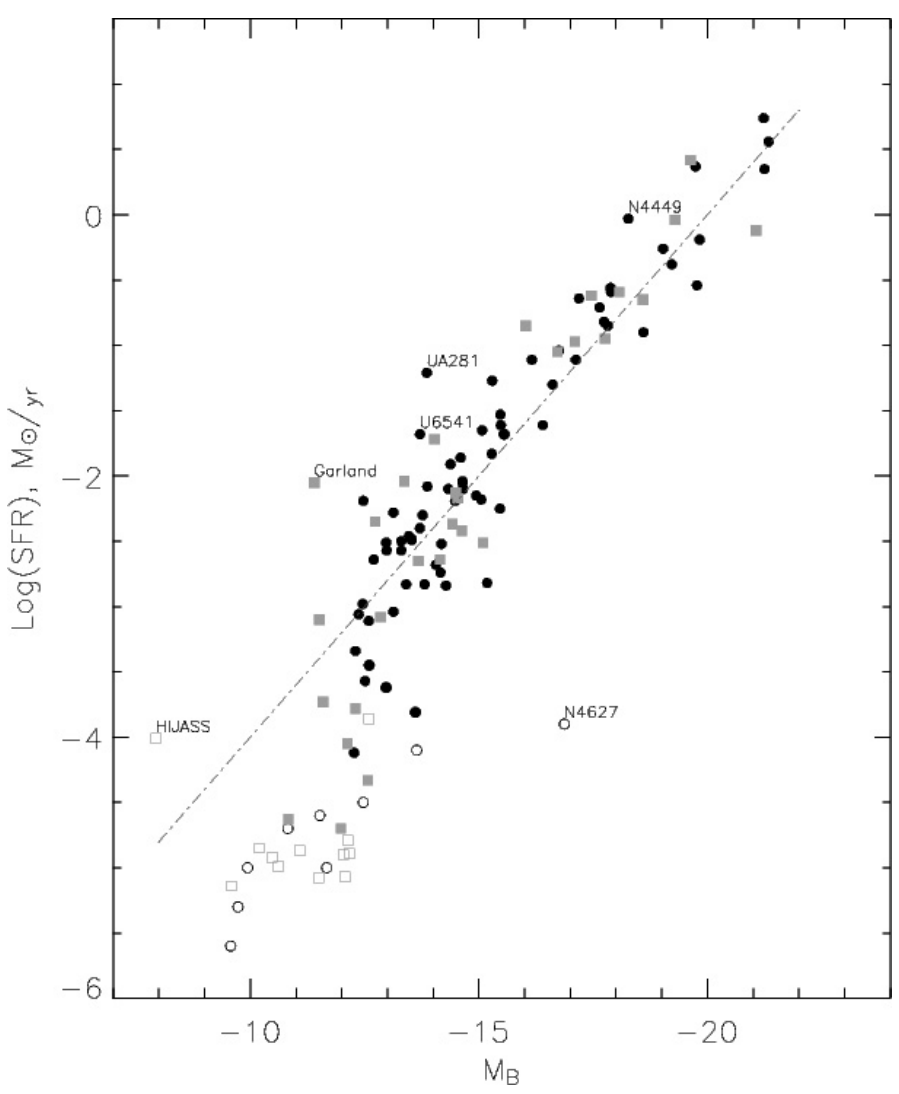

Fig. 2. Star formation rate versus blue absolute magnitude for 78 galaxies in the Canes Venatici I cloud (circles) and 41 members of the M 81 group (squares). The open symbols indicate the galaxies with only an upper limit of their $S F R$. The line corresponds to a constant $S F R$ per unit luminosity.

of low luminosity classified as $T=10$ (=Irr) or 9 (=Im, BCD). Evidently, both these properties are interconnected, being due to the slow rate of the dynamical evolution of the CVnI volume under its low density contrast. At the present time, we have at our disposal a complete set of data on star formation rates and gas reserves in all known members of the cloud. Their $\mathrm{H} \alpha$ images, presented in Fig. 1, show that galaxies of one morphological type possess an enormous variety of emission patterns, which are almost all under the conditions of dynamical isolation $(T I<0)$. This property can be ease understood when the star formation process in galaxies, in particular its rate, is mainly governed by internal but not external mechanisms (tidal triggering).

The distribution of SFR for all 78 galaxies in the CVnI cloud, as a function of their blue absolute magnitude, is presented in Fig. 2 by filled circles. The galaxies with only the upper limit of the $\mathrm{H} \alpha$-flux are given by open circles. For comparison, we include similar data on the complete set of 41 galaxies situated in the nearby virialized group around M 81 (Karachentsev \& Kaisin 2007).

They are shown in this diagram by filled and open squares. As can be seen, the bright members of the cloud and the group follow a common linear relationship $S F R \propto L_{B}$, displayed by the line. The differences between the samples are not large, from either the integrated $S F R$ range or from the scatter of specific $S F R$ per unit luminosity. Faint dwarfs in the two samples with $M_{B}>-13^{m}$ demonstrate a systematic offset from the main sequence. However, their displacement in SFR can be essentially reduced in a new scenario of stellar evolution proposed by Weidner \& Kroupa (2005).

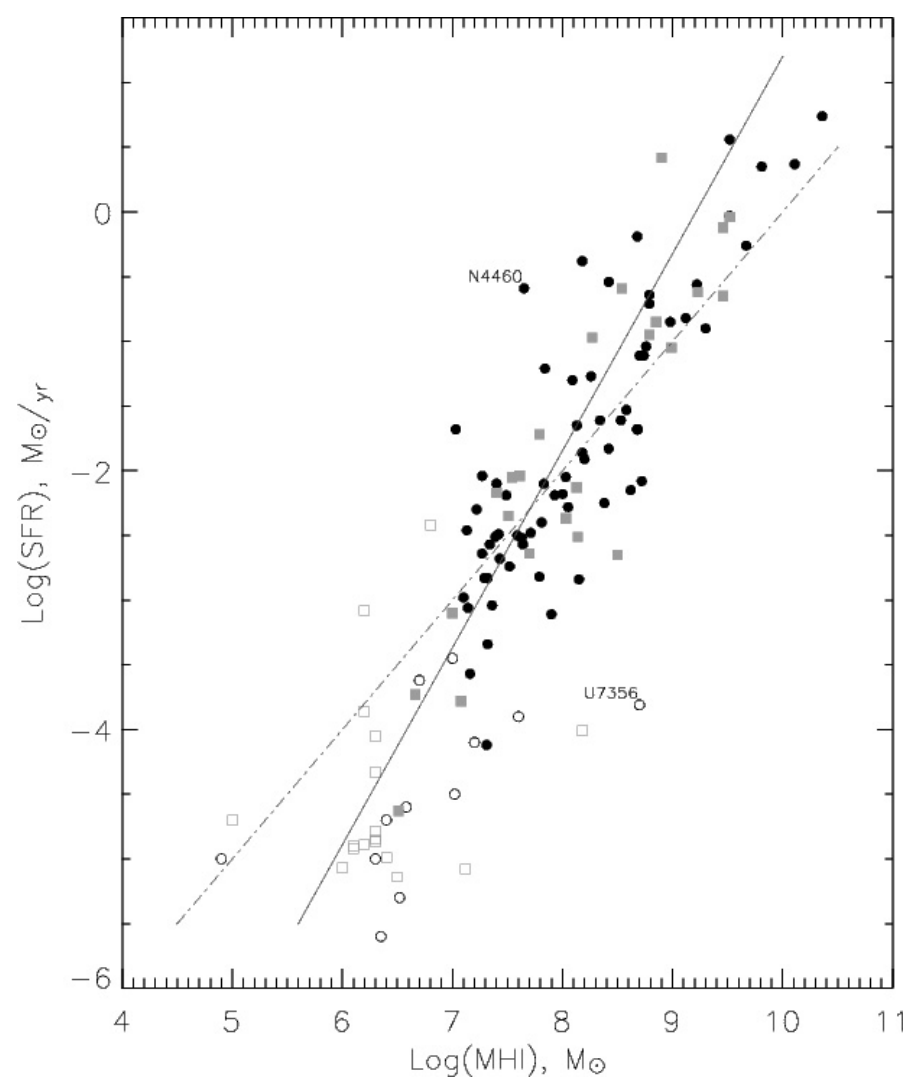

Fig. 3. Star formation rate versus neutral hydrogen mass for galaxies in the CVnI cloud (circles) and the M 81 group (squares). The galaxies with upper limit of $S F R$ or $M_{\mathrm{HI}}$ are indicated by open symbols. The dashed line corresponds to a fixed $S F R$ per unit hydrogen mass and the solid line traces the relationship $S F R \propto M_{\mathrm{HI}}^{1.5}$.

Another important diagram (Fig. 3) illustrates a relationship between $S F R$ and total hydrogen mass $M_{\mathrm{HI}}$ of galaxies. The members of the CVnI cloud, and those of the M 81 group, are shown here by the same symbols as in Fig. 2. As noted by many authors: (Kennicutt 1989, 1998; Taylor \& Webster 2005; Tutukov 2006), spiral and irregular galaxies show a steeper dependence of $S F R$ on $M_{\mathrm{HI}}$ than on luminosity $L_{B}$, namely $S F R \propto$ $M_{\mathrm{HI}}^{1.5}$. This confirms that dIrr galaxies preserve a larger amount of gas than spirals to maintain star formation with the recently observed rates. This diagram also shows that the members of the CVnI cloud and M81 group are mutually well mixed, as in the previous plot.

To estimate the evolutionary status of normal and dwarf galaxies in the CVnI cloud, we used the data on dimensionless parameters in the last two columns of Table 2: $p_{*}=$ $\log \left[(S F R) T_{0} / L_{B}\right]$ and $f_{*}=\log \left[M_{\mathrm{HI}} /(S F R) T_{0}\right]$, which characterize the past and future of the star formation process in a galaxy, on the assumption of permanent star formation rate. The evolutional "past-future" diagram for the observed galaxies is presented in Fig. 4. The members of the CVnI cloud are shown by circles, and the members of the group M 81 are marked by squares. The open symbols correspond to the cases where observations give only the upper limit of the flux in $\mathrm{H} \alpha$ or HI.

The distribution of galaxies in the diagnostic $\left\{p_{*}, f_{*}\right\}$ diagram shows some interesting properties. The members of the CVnI cloud, on the whole, are located quite symmetrically with respect to the origin of coordinates, having median values $p_{*}=+0.02$ and $f_{*}=-0.03$. This means that the observed star formation rates in the $\mathrm{CVnI}$ galaxies proved to be quite 


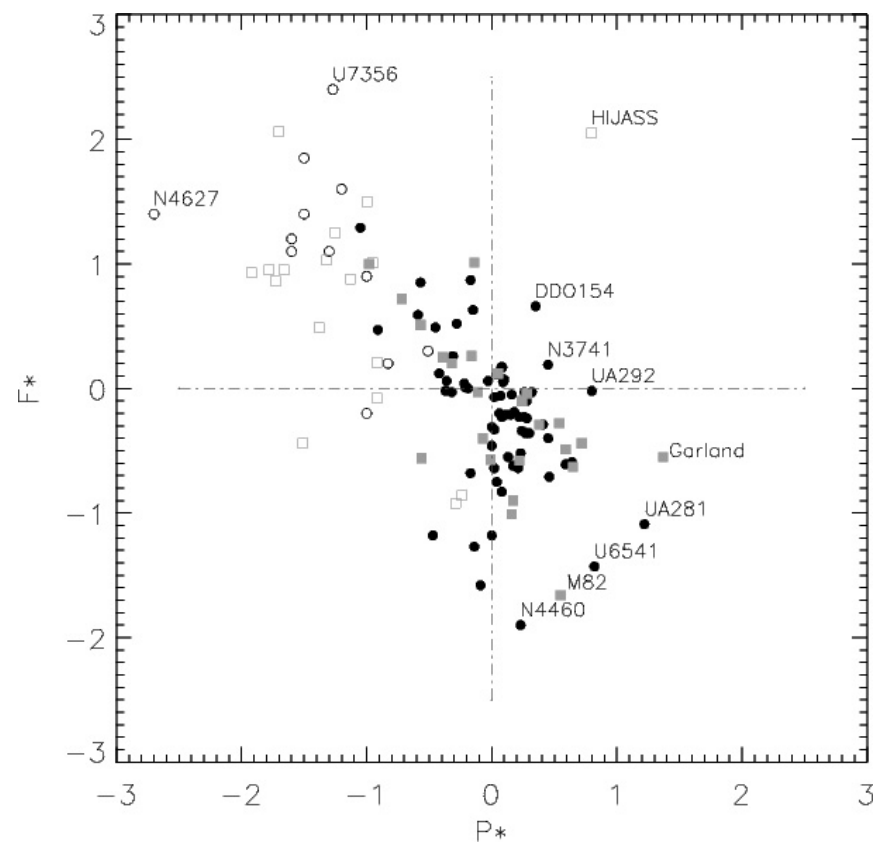

Fig. 4. The CVnI cloud galaxies and the M81 group members on the evolutional plane "past-future": $p_{*}=\log \left([S F R] \cdot T_{0} / L_{B}\right)$ and $f_{*}=\log \left(M_{\mathrm{HI}} /[S F R] \cdot T_{0}\right)$. The galaxies with upper limits of $S F R$ or $M_{\mathrm{HI}}$ are shown by open symbols.

sufficient to reproduce their observed luminosity (baryon mass). Moreover, the cloud galaxies possess sufficient gas reserves to maintain the observed star formation rates during one more Hubble time $T_{0}$, since they are just in the middle of their evolutionary path. For comparison, the median values $p_{*}$ and $f_{*}$ for the M 81 group members are -0.30 and +0.12 , respectively, i.e. their typical value of $S F R$ per unit luminosity is half of that seen in the galaxies of CVnI. However, this difference is easily explicable by the presence of a lot of dSphs around M 81, in which the current star formation can be suppressed by tidal stripping. The CVnI cloud contains only 3 or 4 such "extinct" dwarfs: KK 109, DDO 113, KK 166 and, probably, UGC 8882.

In contrast to spiral galaxies, dIrr galaxies of low luminosity exhibit multiple episodes of global star formation (Dohm-Palmer et al. 2002; Dolphin et al. 2003; Skillman 2005; McConnachie et al. 2005; Young et al. 2007). Stinson et al. (2007) simulated the collapse of isolated dwarf galaxies with the effects of supernova feedback, and showed that star formation in these galaxies occurs in the form of bursts rather than of a sluggish process. The observed $\mathrm{H} \alpha$ flux in galaxies represents its current $S F R$ only over the past 10 Myr (Bell \& Kennicutt 2001; Annibali et al. 2007). The difference between the observed "momentary" and the secular value of $S F R$ averaged over $T_{0}$ will lead to scatter of flashing and dimming dIrr galaxies in the diagram $\left\{p_{*}, f_{*}\right\}$ along the diagonal line $p_{*}=-f_{*}$. This tendency is actually seen in Fig. 4 both for the $\mathrm{CVnI}$ cloud and for the M 81 group members. These data permit us to estimate that the global star formation rate in the dIrrs can vary with time by about an order of magnitude. The most expressive representatives of a dwarf galaxy at a burst stage are UGCA $281=$ Mkn 209 and UGC 6541 = Mkn 178, in which the specific SFR per unit luminosity is 16 times and 7 times higher than the average value, respectively. The known "exploding" galaxy M 82 in the M 81 group has a bit lower specific SFR.

Among 500 galaxies of the Local volume there are only 6 galaxies with a hydrogen mass-to-luminosity ratio greater $5 M_{\odot} / L_{\odot}$. Surprisingly, half of these semi-gaseous galaxies
(DDO 154, NGC 3741 and UGCA 292) reside in the CVnI cloud; all three of them are located in the upper right quadrant of Fig. 4. In contrast Karachentsev \& Kaisin (2007) have found only one peculiar object in the same quadrant of the M 81 group, the dark HI cloud HIJASS with a rather uncertain estimate of SFR. Galaxies probably galaxies start their evolution just from this quadrant, $\left[p_{*}>0, f_{*}>0\right]$, converting their initial gaseous mass into stars.

There is a widespread point of view that close galaxy encounters trigger enhanced star formation in the galaxies. However, Hunter \& Elmegreen (2004) and Noeske et al. (2001) find no correlation between star formation activity in a galaxy and its proximity to other neighboring galaxies. Telles \& Maddox (2000) and James et al. (2004) found that bursting dwarf galaxies inhabit slightly lower density environments than those of denser field. Our data are in agreement with such a conclusion. The left-hand diagram of Fig. 5 presents the distribution of members of the CVnI cloud (circles) and of the M 81 group (squares) versus their specific $S F R$ and tidal index. Here the solid regression line has a slope of $-0.01 \pm 0.03$ corresponding to the galaxies detected in the $\mathrm{H} \alpha$ line, while the dashed regression line with a slope of $-0.07 \pm 0.05$ corresponds to the whole sample of $78+41$ galaxies, including those with upper limits of their $\mathrm{H} \alpha$ flux. This diagram does not show a significant difference in $S F R$ for galaxies in groups $(T I>0)$ as compared to isolated ones. In particular, the strongly disturbed system Garland, near NGC 3077, and the isolated blue galaxy UGCA 281, have almost the same extremely high values of $p_{*}$.

The right diagram of Fig. 5 presents the time of exhaustion of the available reserves of $\mathrm{HI}$ gas, $f_{*}$, for the CVnI cloud and the M 81 group galaxies versus their tidal index. The solid and dashed regression lines with slopes $-0.10 \pm 0.04$ and $-0.01 \pm 0.05$ correspond to the galaxies detected in the $\mathrm{H} \alpha$ and $\mathrm{HI}$ lines, and to the whole sample of galaxies, respectively. Despite the considerable dispersion, a trend of diminishing of the value of $f_{*}$ from isolated galaxies towards interacting ones is seen, which reflects the known tendency of HI-deficiency to rise with increasing density of its environment (Giovanelli \& Haynes 1991).

We regard the isolated $(T I=-0.7)$ lenticular galaxy NGC 4460 to be the most intriguing object in the sample considered. A powerful star formation burst in its center exhausts all available reserve of gas in just 170 Myr. Possibly, we are observing a rare event of interaction of the $\mathrm{S} 0$ galaxy with an intergalactic HI cloud. Such dark, completely starless clouds have already been detected in the nearby groups M 81 (Boyce et al. 2001), Leo-I (Schneider 1985) and the nearby Virgo cluster (Minchin et al. 2005).

\section{Conclusions}

A systematic survey of $\mathrm{H} \alpha$-emission in the nearest scattered cloud $\mathrm{CVnI}$ shows that, in most of its galaxies, the process of active star formation is ongoing despite the low density contrast of this cloud and rather rare interaction between its members. By making full use of our $\mathrm{H} \alpha$-survey, we can estimate the mean density of $S F R$ in the cloud. A conical volume in the distance range from 2 to $10 \mathrm{Mpc}$, resting in a sky region of $\sim 1500$ square degrees, makes $153 \mathrm{Mpc}^{3}$. In this volume we have a summary value $\Sigma(S F R)=18.6 M_{\odot} \mathrm{yr}^{-1}$, which yields an average density of star formation rate $\dot{\rho}_{S F R}=0.12 M_{\odot} \mathrm{yr}^{-1} \mathrm{Mpc}^{-3}$. The obtained value is a little less than $\dot{\rho}_{S F R}=0.165 M_{\odot} \mathrm{yr}^{-1} \mathrm{Mpc}^{-3}$ for a "cell of homogeneity" embracing the group M 81 (Karachentsev \& Kaisin 2007). According to Nakamura et al. (2004), Martin et al. (2005) and Hanish et al. (2006), the average star 

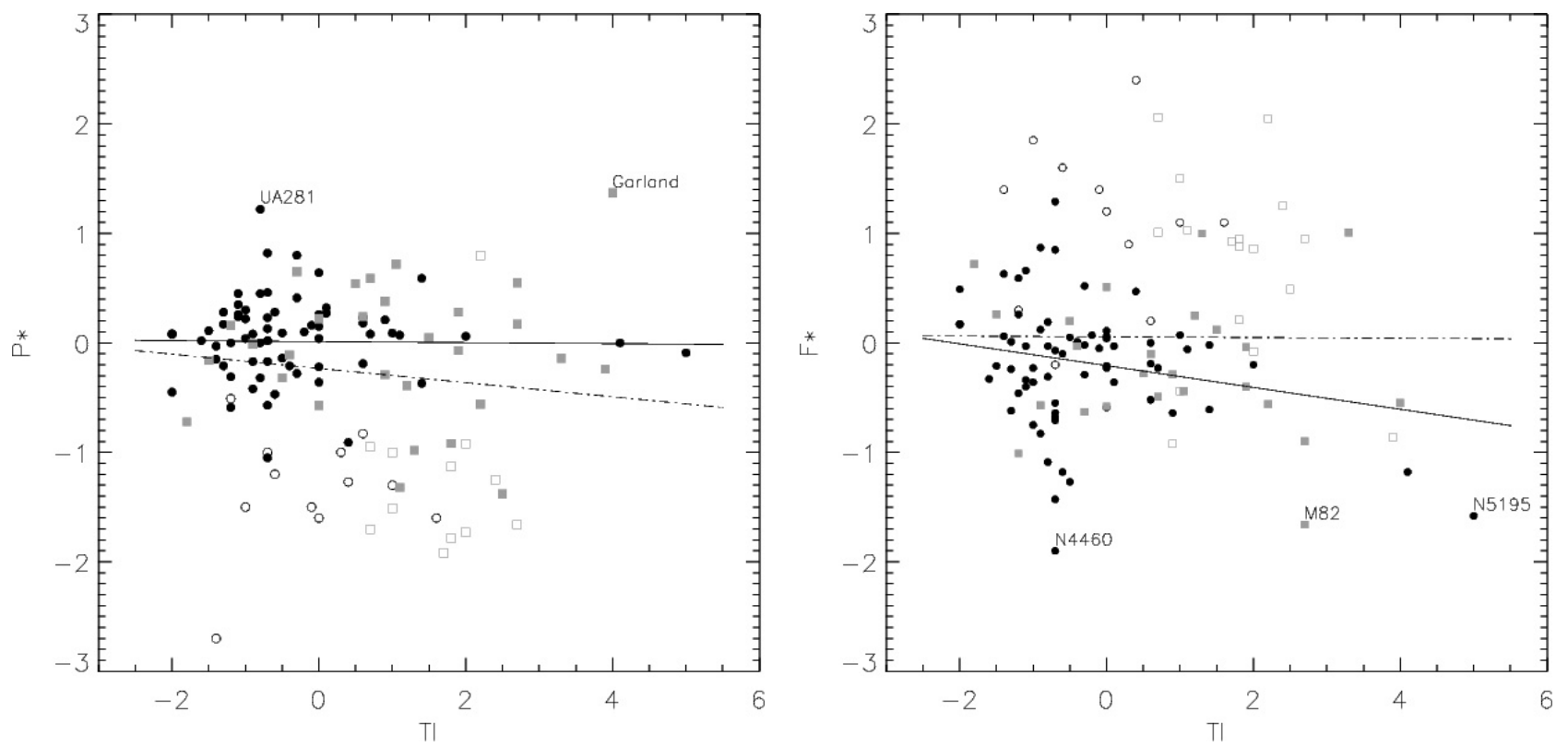

Fig. 5. The specific star formation rate (left) and the time of exhaustion of the available reserves of gas (right) versus the tidal index for the CVnI cloud (circles) and the M 81 group galaxies (squares). The open symbols indicate the galaxies with upper limits of $S F R$ or $M_{\mathrm{HI}}$. The solid and dashed regression lines correspond to the filled only and to the filled plus open symbols, respectively.

formation rate per $1 \mathrm{Mpc}^{3}$ at the present epoch $(z=0)$ is (0.02-0.03) $M_{\odot} \mathrm{yr}^{-1} \mathrm{Mpc}^{-3}$. Consequently, the CVnI cloud has an excess of SFR density 4-6 times higher in comparison with the mean global quantity, which roughly corresponds to the cloud density contrast on the sky, $\Delta N / N \sim 4$. From this we conclude that the CVnI cloud is characterized by a usual norm of star formation in its galaxies.

Acknowledgements. We would like to thank Margarita Sharina for her help with the photometry of UGCA 281 . We are also grateful to B. Tully for useful discussions. Support associated with HST program 10905 was provided by NASA through a grant from the Space Telescope Science Institute, which is operated by the Association of Universities for Research in Astronomy, Inc., under NASA contract NAS5-26555. This work was also supported by RFFI grant 07-02-00005 and grant DFG-RFBR 06-02-04017.

\section{References}

Afanasiev, V. L., Gazhur, E. B., Zhelenkov, S. R., \& Moiseev, A. V. 2005, Bull. $\mathrm{SAO}, 58,90$

Annibali, F., Aloisi, A., Mack, J., et al. 2007 [arXiv: 0708. 0852]

Arp, H. 1966, Atlas of Peculiar Galaxies, ApJS, 14, 1

Begum, A., \& Chengalur, J. N. 2005, MNRAS, 362, 609

Begum, A., Chengalur, J. N., Karachentsev, I. D., Kaisin, S. S., \& Sharina, M. E. 2006, MNRAS, 365, 1220

Bell, E. F., \& Kennicutt, R. C. 2001, ApJ, 548, 681

Boyce, P. J., Minchin, R. F., Kilborn, V. A., et al. 2001, ApJ, 560, L127

de Vaucouleurs, G. 1975, in Galaxies and the Universe, ed. A. Sandage, M. Sandage, \& J. Kristian (Chicago, Univ. of Chicago Press), 557

de Vaucouleurs, G., de Vaucouleurs, A., Corwin, A., et al. 1991, Third Reference Catalogue of Bright Galaxies (New-York: Springer-Verlag)

Dohm-Palmer, R. C., Skillman, E. D., Mater, M., et al. 2002, AJ, 123, 813

Dolphin, A. E., Saha, A., Skillman, E. D., et al. 2003, AJ, 126, 187

Gallagher, J. S., Hunter, D. A., \& Tutukov, A. V. 1984, ApJ, 284, 544

Gil de Paz Madore, B. F., \& Pevunova, O. 2003, ApJS, 147, 29

Giovanelli, R., \& Haynes, M. P. 1991, ARA\&A, 29, 499

Hanish, D. J., Meurer, G. R., Ferguson, H. C., et al. 2006, ApJ, 649, 150

Hodge, P. W., \& Kennicutt, R. C. 1983, AJ, 88, 296

Hoopes, C. G., Walterbos, R. A. M., \& Rand, R. J. 1999, ApJ, 522, 669

Huchtmeier, W. K., Karachentsev, I. D., Karachentseva, V. E., \& Ehle, M. 2000, A\&AS, 141, 469

Huchtmeier, W. K., Karachentsev, I. D., \& Karachentseva, V. E. 2003, A\&A, 401,483
Hunter, D. A., \& Elmegreen, B. G. 2004, AJ, 128, 2170

Hunter, D. A., \& Gallagher, J. S. 1992, ApJ, 391, L9

James, P. A., Shane, N. S., Beckman, J. E., et al. 2004, A\&A, 414, 23

Karachentsev, I. D. 1966, Astrofizika, 2, 81

Karachentsev, I. D., \& Kaisin, S. S. 2007, AJ, 133, April

Karachentsev, I. D., Sharina, M. E., \& Dolphin, A. E. 2003, A\&A, 398, 467

Karachentsev, I. D., Karachentseva, V. E., Huchtmeier, W. K., \& Makarov, D. I. 2004, AJ, 127, 2031

Karachentsev, I. D., Dolphin, A. E., \& Tully, R. B. 2006, AJ, 131, 1361

Kennicutt, R. C. 1998, ApJ, 498, 541

Kennicutt, R. C. 1989, ApJ, 344, 685

Kennicutt, R. C., Edgar, B. K., \& Hodge, P. W. 1989, ApJ, 337, 761

Makarova, L. N., Karachentsev, I. D., \& Georgiev, T. B. 1997, Astron. Lett., 23, 435

Martin, D. C., Seibert, M., Buat, V., et al. 2005, ApJ, 619, 59

McConnachie, A. W., Arimoto, N., Irwin, M., \& Tolstoy, E. 2006, MNRAS, 373 , 715

Miller, B. W., \& Hodge, P. 1994, ApJ, 427, 656

Minchin, R. F., Davies, J., Disney, M., et al. 2005, ApJ, 622, L21

Nakamura, O., Fukugita, M., et al. 2004, AJ, 127, 2511

Oke, J. B. 1990, AJ, 99, 1621

Papaderos, P., et al. 1996, A\&AS, 120, 207

Rekola, R., Jerjen, H., \& Flynn, C. 2005, A\&A, 437, 823

Sargent, W. L. W., \& Searle, L. 1970, ApJ, 162, L155

Seth, A. C., Dalcanton, J. J., \& de Jong, R. S. 2005, AJ, 129, 1331

Schlegel, D. J., Finkbeiner, D. P., \& Davis, M. 1998, ApJ, 500, 525

Schneider, S. E. 1985, ApJ, 288, L33

Skillman, E. D. 2005, New Astron. Rev., 49, 453

Spergel, D. N., Verde, L., Peiris, H. V., et al. 2003, ApJS, 148, 175

Stinson, G. S., Dalcanton, J. J., Quinn, T., et al. 2007, ApJ, 667, 170

Strickland, D. K., Heckman, T. M., Colbert, E. J. M., et al. 2004, ApJ, 606, 829

Taylor, E. N., \& Webster, R. L. 2005, ApJ, 634, 1067

Telles, E. \& Maddox, S. 2000, MNRAS, 311, 307

Tonry, J. L., Dressler, A., Blakeslee, J. P., et al. 2001, ApJ, 546, 681

Tully, R. B. 1988, Nearby Galaxy Catalog (Cambridge Univ. Press)

Tully, R. B., Shaya, E. J., \& Pierce, M. J. 1992, ApJS, 80, 479

Tully, R. B., et al. 2007, in preparation

Tutukov, A. V. 2006, Astron. Rep., 50, 526

van Zee, L. 2000, AJ, 119, 2757

Vennik, J. 1984, Tartu Astron. Obs. Publ., 73, 1

Verheijen, M. A. W. 2001, ApJ, 563, 694

Weidner, C., \& Kroupa, P. 2005, ApJ, 625, 754

Young, J. S., Allen, L., Kenney, J. D., \& Rownd, B. 1996, AJ, 112, 1903

Young, L. M., Skillman, E. D., Weisz, D. R., \& Dolphin, A. E. 2007, ApJ, 659, 331 